\title{
Synthesis of the Alleged Structures of Fortisterol and Herbarulide and Structural Revision of Herbarulide
}

\author{
Fenja L. Duecker, Robert C. Heinze, Mira Mueller, Sudong Zhang, Philipp Heretsch* \\ Institute of Chemistry and Biochemistry, Freie Universität Berlin, Takustraße 3, 14195 Berlin, \\ Germany \\ *Correspondence to: philipp.heretsch@fu-berlin.de
}

\section{Supporting Information}

Table of Contents

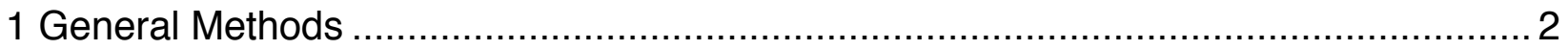

2 Experimental Procedures and Characterization Data............................................. 3

2.1 Synthesis of Burawoy Ketone (8) and Initial Radical Rearrangement ..................... 3

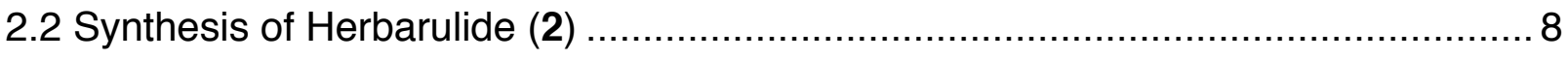

2.3 Synthesis of (22E)-5,6-Epoxy-5,6-secocampesta-4,7,22-triene-3,6-dione (1) ...... 10

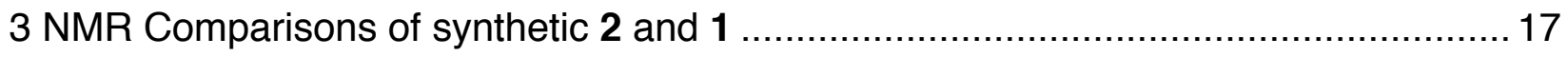

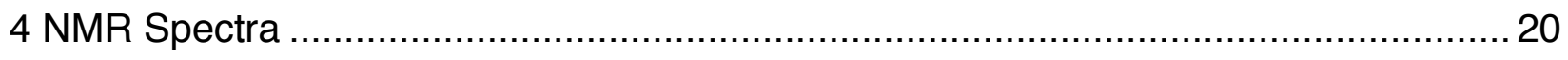

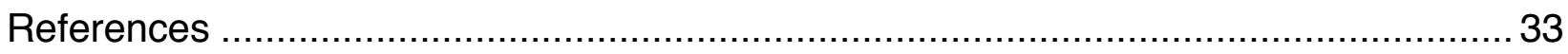




\section{General Methods}

All reactions sensitive to moisture and/or air were carried out using heat gun-dried glassware, an argon atmosphere, and anhydrous solvents. Anhydrous dichloromethane, toluene, and $\mathrm{Et}_{2} \mathrm{O}$ were taken from a $\mathrm{M}$. Braun GmbH MB SPS-800 solvent purification system. THF was distilled from sodium and stored over $4 \AA$ molecular sieves. Ethyl acetate and nhexane were purified by distillation on a rotary evaporator. All other solvents and commercially available reagents were used without further purification unless otherwise stated. In case reactions required heating, this was carried out using silicone oil baths. Reactions were monitored by thin-layer chromatography (TLC) carried out on Merck Silica Gel 50 F245-plates and visualized by fluorescence quenching under UV-light or an aqueous solution of cerium sulfate and phosphomolybdic acid and heat as developing agent. Column chromatographic purification was performed on Macherey-Nagel Silica Gel 60 M (40-60 $\mu \mathrm{m})$. HPLC separation was performed on a high-pressure gradient system (stainless steel), equipped with a Shimadzu LC-8A pump, Shimadzu CBM-20A controller, variable wavelength UV detector from Knauer and a Rheodyne injector with $10 \mathrm{~mL}$ sample loop. Concentration under reduced pressure was performed by rotary evaporation at $45^{\circ} \mathrm{C}$ and appropriate pressure, followed by exposure to high vacuum (10-3 mbar) at $25^{\circ} \mathrm{C}$. NMR spectra were recorded on either a Jeol ECP500 (500 MHz), a Bruker AVANCE III $500(500 \mathrm{MHz})$ or a Varian INOVA600 $(600 \mathrm{MHz})$. Chemical shifts $\delta$ are reported in parts per million $(\mathrm{ppm})$ and are referenced using residual undeuterated solvent $\left(\mathrm{CDCl}_{3}: \delta_{\mathrm{H}}=7.26 \mathrm{ppm}, \delta_{\mathrm{C}}\right.$ $=77.16$ ppm; DMSO- $\mathrm{d}_{6}: \delta_{\mathrm{H}}=2.50 \mathrm{ppm}, \delta_{\mathrm{C}}=39.52 \mathrm{ppm}$; pyridine- $\mathrm{d}_{5}: \delta_{\mathrm{H}}=8.74 \mathrm{ppm}, \delta_{\mathrm{C}}=150.35 \mathrm{ppm}$; unless otherwise stated) as an internal reference at $298 \mathrm{~K}$. The given multiplicities are phenomenological; thus, the actual appearance of the signals is stated and not the theoretically expected one. The following abbreviations are used to designate multiplicities: $s=$ singlet, $d=$ doublet, $t=$ triplet, $q=$ quartet, $p=$ pentet, $\mathrm{br}=$ broad, and combinations thereof. In case no multiplicity could be identified, the chemical shift range of the signal is given ( $m=$ multiplet). Overlaid signals (typically by solvent) are marked as ov. Infrared (IR) spectra were measured on a Jasco FT/IR-4100 Type A spectrometer with a TGS detector. Wavenumbers $\tilde{v}$ are given in $\mathrm{cm}^{-1}$ and intensities are as follows: $\mathrm{s}=$ strong, $\mathrm{m}=$ medium, $\mathrm{w}=$ weak. High-resolution mass spectra (HRMS) were recorded using an Agilent 6210 ESI-TOF or an lonspec QFT-7 ESI-TOF spectrometer. Optical rotations were measured on a JASCO P-2000 polarimeter at $589 \mathrm{~nm}$ using $100 \mathrm{~mm}$ cells and the solvent and concentration $(\mathrm{g} / 100 \mathrm{~mL})$ indicated. Melting points were measured on a Stuart SMP30. 


\section{Experimental Procedures and Characterization Data}

\subsection{Synthesis of Burawoy Ketone (8) and Initial Radical Rearrangement}

(22E)-5a,6a-Epoxyergosta-7,22-dien-3ß-ol (S1)
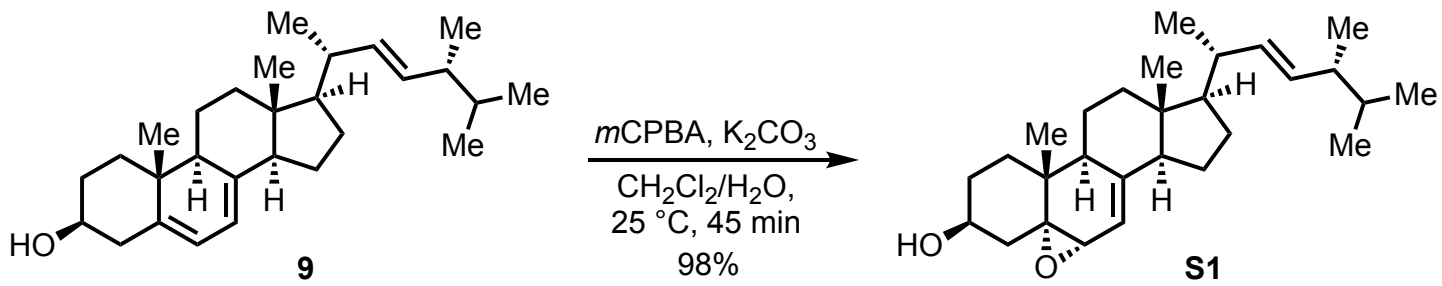

To a suspension of (-)-ergosterol (9) $\left(5.00 \mathrm{~g}, 12.6 \mathrm{mmol}, 1.0\right.$ eq.) in $\mathrm{CH}_{2} \mathrm{Cl}_{2}$ (125 mL) was added a solution of $\mathrm{K}_{2} \mathrm{CO}_{3}\left(3.48 \mathrm{~g}, 25.3 \mathrm{mmol}, 2.0\right.$ eq.) in $\mathrm{H}_{2} \mathrm{O}(125 \mathrm{~mL})$. In a separate flask, $m$ CPBA $(70 \% \mathrm{w} / \mathrm{w}, 3.10 \mathrm{~g}$, $12.6 \mathrm{mmol}, 1.0$ eq.) was suspended in $\mathrm{CH}_{2} \mathrm{Cl}_{2}(50 \mathrm{~mL})$ by sonication to give a turbid solution, which was then added dropwise to the reaction mixture over a period of $5 \mathrm{~min}$. After vigorous stirring for $45 \mathrm{~min}$ at $25^{\circ} \mathrm{C}$ the phases were separated, and the aqueous phase was extracted with $\mathrm{CH}_{2} \mathrm{Cl}_{2}(3 \times 75 \mathrm{~mL})$. The combined organic phases were sequentially washed with $\mathrm{NaHCO}_{3}$ (sat. aq., $250 \mathrm{~mL}$ ) and brine (sat., 250 $\mathrm{mL}$ ), dried over $\mathrm{MgSO}_{4}$, and the solvent was removed under reduced pressure to give epoxide $\mathbf{S 1}$ (5.10 g, $12.4 \mathrm{mmol}, 98 \%$ ) as a white solid, which was used in the next step without further purification.

M.p.: $\quad 233-235^{\circ} \mathrm{C}\left(\mathrm{CHCl}_{3}\right)$.

TLC: $\quad R_{\mathfrak{f}}=0.40($ nhexane/EtOAc 1:1).

1H-NMR: $\quad\left(500 \mathrm{MHz}, \mathrm{CDCl}_{3}\right) ; \delta[\mathrm{ppm}]=5.45-5.41(\mathrm{~m}, 1 \mathrm{H}), 5.24-5.12(\mathrm{~m}, 2 \mathrm{H}), 4.01-3.91(\mathrm{~m}$, $1 \mathrm{H}), 3.00(\mathrm{~d}, J=4.1 \mathrm{~Hz}, 1 \mathrm{H}), 2.26(\mathrm{dd}, J=13.0,11.5 \mathrm{~Hz}, 1 \mathrm{H}), 2.06-1.99(\mathrm{~m}, 3 \mathrm{H}), 1.99-$ $1.92(\mathrm{~m}, 2 \mathrm{H}), 1.87-1.80(\mathrm{~m}, 2 \mathrm{H}), 1.75-1.66(\mathrm{~m}, 2 \mathrm{H}), 1.57-1.50(\mathrm{~m}, 3 \mathrm{H}), 1.51-1.38$ (m, 3H), $1.35-1.20(\mathrm{~m}, 4 \mathrm{H}), 1.02(\mathrm{~s}, 3 \mathrm{H}), 1.01(\mathrm{~d}, J=6.6 \mathrm{~Hz}, 3 \mathrm{H}), 0.91(\mathrm{~d}, J=6.9 \mathrm{~Hz}$, $3 \mathrm{H}), 0.83(\mathrm{~d}, J=6.8 \mathrm{~Hz}, 3 \mathrm{H}), 0.82(\mathrm{~d}, J=6.8 \mathrm{~Hz}, 3 \mathrm{H}), 0.55$ (s, 3H).

13C-NMR: $\quad\left(126 \mathrm{MHz}, \mathrm{CDCl}_{3}\right) ; \delta[\mathrm{ppm}]=148.1,135.5,132.2,114.9,68.5,67.0,55.8,55.0,54.7,42.9$, $42.3,41.5,40.5,39.9,38.8,35.1,33.6,33.2,31.3,28.0,23.3,21.2,21.1,20.1,19.8,17.7$, $16.5,12.2$.

IR: $\quad\left(\right.$ neat); $\tilde{v}\left[\mathrm{~cm}^{-1}\right]=3441(\mathrm{br} \mathrm{w}), 2953(\mathrm{~s}), 2871(\mathrm{~m}), 1457(\mathrm{w}), 1370(\mathrm{w}), 1052(\mathrm{w}), 969(\mathrm{w})$, $902(w), 870(w)$.

HRMS: (ESI-TOF); $m / z$ calcd. for $\mathrm{C}_{28} \mathrm{H}_{44} \mathrm{O}_{2} \mathrm{Na}^{+}[\mathrm{M}+\mathrm{Na}]^{+}:$435.3234, found: 435.3252.

Opt. Act:: $[a]_{D}^{22}=-66.5\left(c=1.00, \mathrm{CHCl}_{3}\right)$.

All characterization data were consistent with those reported in the literature $\left({ }^{1} \mathrm{H}\right.$ - and $\left.{ }^{13} \mathrm{C}-\mathrm{NMR}, \mathrm{IR}\right) .{ }^{[1]}$ 

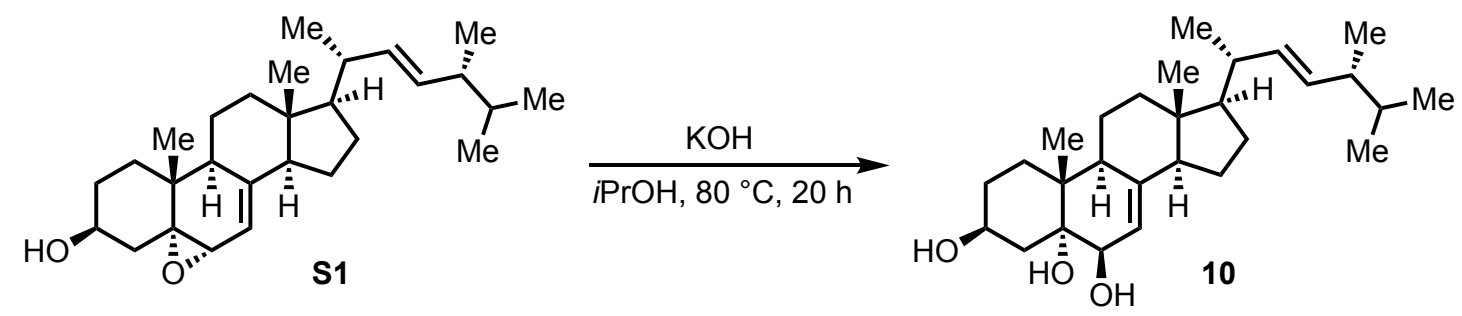

To epoxide S1 (5.10 g, $12.4 \mathrm{mmol}, 1.0$ eq.) were added $\mathrm{KOH}$ ( $1 \mathrm{M} \mathrm{in} \mathrm{H}_{2} \mathrm{O}, 50 \mathrm{~mL}$ ) and $\mathrm{PrOH}(50 \mathrm{~mL})$. The suspension was heated to $80^{\circ} \mathrm{C}$ and stirred at this temperature for $18 \mathrm{~h}$. The reaction mixture was allowed to cool to $25^{\circ} \mathrm{C}$ and neutralized with $\mathrm{HCl}\left(1 \mathrm{M} \mathrm{in} \mathrm{H}_{2} \mathrm{O}\right)$. The layers were separated, and the aqueous phase was extracted with $\mathrm{CHCl}_{3} / \mathrm{PrOH}(4: 1,3 \times 75 \mathrm{~mL})$. The combined organic phases were washed with brine (sat., $250 \mathrm{~mL}$ ), dried over $\mathrm{MgSO}_{4}$, and the solvent was removed under reduced pressure to give crude triol 10 (5.33 g, $12.4 \mathrm{mmol}$, quant.) as a light yellow solid, which was used in the next step without further purification.

Analytically pure triol $\mathbf{1 0}$ could be obtained by column chromatography (silica gel, EtOAc) as a colorless solid.

M.p.: $\quad 231-233^{\circ} \mathrm{C}\left(\mathrm{CHCl}_{3}\right)$.

TLC: $\quad R_{\mathrm{f}}=0.25(\mathrm{EtOAC})$.

1H-NMR: $\quad\left(500 \mathrm{MHz}, \mathrm{DMSO}-\mathrm{d}_{6}\right) ; \delta[\mathrm{ppm}]=5.23(\mathrm{dd}, J=15.3,7.2 \mathrm{~Hz}, 1 \mathrm{H}), 5.17(\mathrm{dd}, J=15.3,8.0 \mathrm{~Hz}$, $1 \mathrm{H}), 5.10-5.06(\mathrm{~m}, 1 \mathrm{H}), 4.49(\mathrm{~d}, J=5.5 \mathrm{~Hz}, 1 \mathrm{H}), 4.22(\mathrm{~d}, J=5.7 \mathrm{~Hz}, 1 \mathrm{H}), 3.81-3.72(\mathrm{~m}$, $1 \mathrm{H}), 3.58(\mathrm{~s}, 1 \mathrm{H}), 3.39-3.35(\mathrm{~m}, 1 \mathrm{H}), 2.04-1.91(\mathrm{~m}, 3 \mathrm{H}), 1.90-1.77(\mathrm{~m}, 3 \mathrm{H}), 1.70-$ $1.57(\mathrm{~m}, 2 \mathrm{H}), 1.52-1.43(\mathrm{~m}, 5 \mathrm{H}), 1.42-1.34(\mathrm{~m}, 2 \mathrm{H}), 1.31-1.21(\mathrm{~m}, 5 \mathrm{H}), 0.99(\mathrm{~d}, J=$ $6.5 \mathrm{~Hz}, 3 \mathrm{H}), 0.91(\mathrm{~s}, 3 \mathrm{H}), 0.89(\mathrm{~d}, J=6.9 \mathrm{~Hz}, 3 \mathrm{H}), 0.81(\mathrm{~d}, J=6.6 \mathrm{~Hz}, 3 \mathrm{H}), 0.80$ (d, J = 6.6 $\mathrm{Hz}, 3 \mathrm{H}), 0.54(\mathrm{~s}, 3 \mathrm{H})$.

13C-NMR: $\quad\left(126 \mathrm{MHz}, \mathrm{DMSO}-\mathrm{d}_{6}\right) ; \delta[\mathrm{ppm}]=139.6,135.4,131.4,119.5,74.5,72.1,66.5,55.3,54.2$, $43.0,42.3,42.0,40.2,40.1,39.0,36.6,32.5$ (2C), 31.2, 27.7, 22.1, 21.3, 21.0, 19.7, 19.4, $17.7,17.3,12.0$.

IR: $\quad\left(\right.$ neat); $\tilde{v}\left[\mathrm{~cm}^{-1}\right]=3364(\mathrm{br} \mathrm{w}), 2956(\mathrm{~s}), 2928(\mathrm{~s}), 2871(\mathrm{~m}), 1722(\mathrm{~m}), 1457(\mathrm{~m}), 1372(\mathrm{w})$, $1269(\mathrm{~m}), 1050(\mathrm{w}), 973(\mathrm{~m})$.

HRMS: $\quad$ (ESI-TOF); $\mathrm{m} / \mathrm{z}$ calcd. for $\mathrm{C}_{28} \mathrm{H}_{46} \mathrm{O}_{3} \mathrm{Na}^{+}[\mathrm{M}+\mathrm{Na}]+:$ 453.3339, found: 453.3359 .

Opt. act. $\quad[\mathrm{a}]_{\mathrm{D}}^{20}=-56.3\left(c=1.00,\left(\mathrm{CH}_{3}\right)_{2} \mathrm{SO}\right)$.

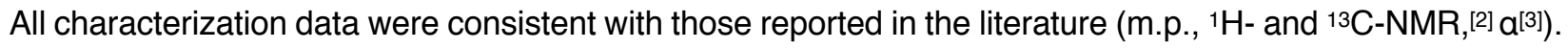



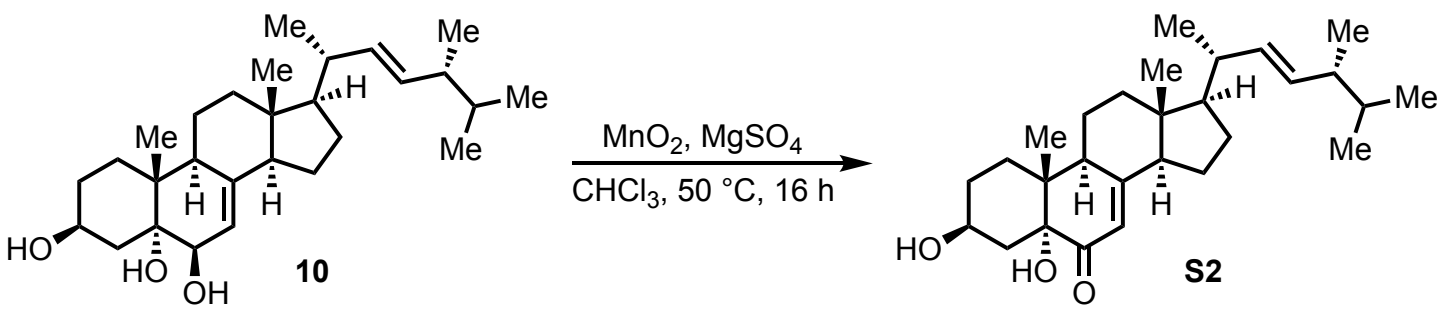

To a stirred suspension of triol 10 (5.33 g, $12.4 \mathrm{mmol}, 1.0$ eq.) in $\mathrm{CHCl}_{3}\left(125 \mathrm{~mL}\right.$ ) were added $\mathrm{MgSO}_{4}$ $(1.25 \mathrm{~g})$ and $\mathrm{MnO}_{2}\left(8.58 \mathrm{~g}, 98.8 \mathrm{mmol}, 8.0\right.$ eq.). The resulting mixture was heated to $50^{\circ} \mathrm{C}$ and stirred at this temperature for $16 \mathrm{~h}$. The reaction mixture was allowed to cool to $25^{\circ} \mathrm{C}$ and $\mathrm{CHCl}_{3} / \mathrm{PrOH}(4: 1,65 \mathrm{~mL})$ and silica gel were added. The solvent was removed under reduced pressure and the residue was added onto a short plug of silica gel $(10 \mathrm{~cm})$ to remove manganese solids. It was rinsed with $\mathrm{CHCl}_{3} / \mathrm{PrOH}(4: 1)$ until all product was recovered from the column to give a mix of crude a-ketol $\mathbf{S 2}$ along with over oxidized side product 7 as a yellow solid $(5.3 \mathrm{~g})$.

Analytically pure a-ketol $\mathbf{S} 2$ could be obtained by column chromatography (silica gel, nhexane/EtOAc 1:2) as a colorless solid.

M.p.: $\quad 248-250^{\circ} \mathrm{C}(\mathrm{EtOAC})$.

TLC: $\quad R_{\mathrm{f}}=0.50(\mathrm{EtOAc})$.

1H-NMR: $\quad\left(500 \mathrm{MHz}\right.$, pyridine- $\left.\mathrm{d}_{5}\right) ; \delta[\mathrm{ppm}]=7.23(\mathrm{~s}, 1 \mathrm{H}), 6.24-6.11(\mathrm{~m}, 1 \mathrm{H}), 5.94(\mathrm{t}, \mathrm{J}=2.3 \mathrm{~Hz}$, $1 \mathrm{H}), 5.29$ (dd, $J=15.2,7.7 \mathrm{~Hz}, 1 \mathrm{H}), 5.21(\mathrm{dd}, J=15.3,8.5 \mathrm{~Hz}, 1 \mathrm{H}), 4.76-4.66(\mathrm{~m}, 1 \mathrm{H})$, $3.02-2.91(\mathrm{~m}, 2 \mathrm{H}), 2.33(\mathrm{dd}, J=13.8,11.4 \mathrm{~Hz}, 1 \mathrm{H}), 2.27-2.18(\mathrm{~m}, 2 \mathrm{H}), 2.09-2.00(\mathrm{~m}$, $3 \mathrm{H}), 1.95-1.87(\mathrm{~m}, 1 \mathrm{H}), 1.87-1.81(\mathrm{~m}, 1 \mathrm{H}), 1.79-1.74(\mathrm{~m}, 1 \mathrm{H}), 1.74-1.68(\mathrm{~m}, 2 \mathrm{H})$, 1.62 (qd, $J=13.4,4.3 \mathrm{~Hz}, 1 \mathrm{H}), 1.54-1.45(\mathrm{~m}, 3 \mathrm{H}), 1.41-1.34(\mathrm{~m}, 1 \mathrm{H}), 1.33-1.23(\mathrm{~m}$, 2H), $1.12(\mathrm{~s}, 3 \mathrm{H}), 1.08$ (d, $J=6.6 \mathrm{~Hz}, 3 \mathrm{H}), 0.99$ (d, $J=6.8 \mathrm{~Hz}, 3 \mathrm{H}), 0.90$ (d, $J=4.3 \mathrm{~Hz}$, $3 \mathrm{H}), 0.89(\mathrm{~d}, J=4.4 \mathrm{~Hz}, 3 \mathrm{H}), 0.63(\mathrm{~s}, 3 \mathrm{H})$.

13C-NMR: $\quad\left(126 \mathrm{MHz}\right.$, pyridine- $\left.d_{5}\right) ; \delta[p p m]=200.4,164.4,136.3,132.9,121.1,77.8,67.5,56.5,56.3$, $45.1,44.8,43.6,41.7,41.1$, 39.7, 38.2, 33.8, 32.2, 31.7, 28.7, 23.3, 22.6, 21.8, 20.6, 20.3, $18.3,16.9,13.2$.

IR: $\quad\left(\right.$ neat); $\tilde{v}\left[\mathrm{~cm}^{-1}\right]=3485(\mathrm{br} \mathrm{w}), 3320$ (br w), $2954(\mathrm{~s}), 2871(\mathrm{~m}), 1738(\mathrm{w}), 1673(\mathrm{~s}), 1623$ $(\mathrm{w}), 1458(\mathrm{~m}), 1371(\mathrm{~m}), 1250(\mathrm{~m}), 1155(\mathrm{~m}), 1081(\mathrm{~m}), 970(\mathrm{~m}), 870(\mathrm{~m}), 827(\mathrm{w})$.

HRMS: $\quad$ (ESI-TOF); $\mathrm{m} / z$ calcd. for $\mathrm{C}_{28} \mathrm{H}_{44} \mathrm{O}_{3} \mathrm{Na}^{+}[\mathrm{M}+\mathrm{Na}]^{+}: 451.3183$, found: 451.3177.

Opt. act. $[\mathrm{a}]_{\mathrm{D}}^{20}=+8.8\left(c=1.00, \mathrm{CHCl}_{3}\right)$. 
All characterization data were consistent with those reported in the literature (IR, ${ }^{\mathrm{H}} \mathrm{H}-\mathrm{NMR}, \mathrm{a}$ and $\mathrm{m} . \mathrm{p},{ }^{[4]}{ }^{13 \mathrm{C}-}$ NMR[5]).

(22E)-5a-Hydroxyergosta-7,22-dien-6-on-3ß-yl acetate/Burawoy ketone (8)
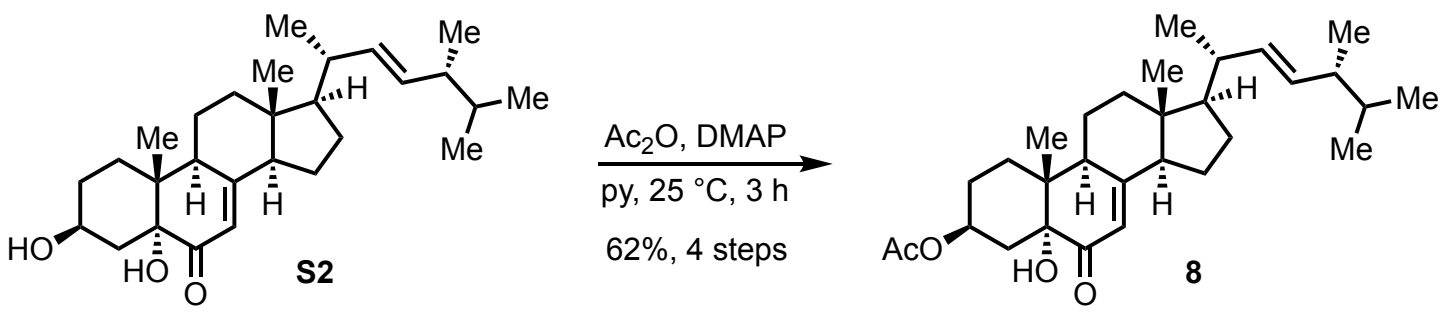

To a stirred solution of crude a-ketol $\mathbf{S} 2(5.3 \mathrm{~g}, 12.4 \mathrm{mmol}, 1.0$ eq.) in pyridine $(50 \mathrm{~mL})$ were added 4dimethylaminopyridine (150 mg, $1.23 \mathrm{mmol}, 0.1$ eq.) and acetic anhydride ( $3.3 \mathrm{~mL}, 34 \mathrm{mmol}, 2.75$ eq.) at $25^{\circ} \mathrm{C}$ and stirring was continued at this temperature for $3 \mathrm{~h}$. The reaction mixture was poured into $\mathrm{H}_{2} \mathrm{O}$ $(50 \mathrm{~mL})$ and the phases were separated. The aqueous phase was extracted with $\mathrm{CH}_{2} \mathrm{Cl}_{2}(3 \times 75 \mathrm{~mL})$ and the combined organic phases were washed with $\mathrm{HCl}\left(1 \mathrm{M} \mathrm{in} \mathrm{H}_{2} \mathrm{O}, 3 \times 200 \mathrm{~mL}\right)$ and brine (sat., $400 \mathrm{~mL}$ ), dried over $\mathrm{MgSO}_{4}$, and the solvent was removed under reduced pressure to give a yellow residue $(5.7 \mathrm{~g})$. Recrystallization of the crude product from EtOAc gave Burawoy ketone (8) (3.68 g, $7.80 \mathrm{mmol}$, 62\% over 4 steps) as colorless needles.

M.p.: $\quad 261-264^{\circ} \mathrm{C}(\mathrm{EtOAc})$.

TLC: $\quad R_{\mathrm{f}}=0.35($ nhexane/EtOAc $3: 1)$.

1H-NMR: $\quad\left(500 \mathrm{MHz}, \mathrm{CDCl}_{3}\right) ; \delta[\mathrm{ppm}]=5.63(\mathrm{t}, J=2.3 \mathrm{~Hz}, 1 \mathrm{H}), 5.23(\mathrm{dd}, J=15.2,7.7 \mathrm{~Hz}, 1 \mathrm{H})$, 5.14 (dd, $J=15.3,8.2 \mathrm{~Hz}, 1 \mathrm{H}$ ), $5.11-5.04(\mathrm{~m}, 1 \mathrm{H}), 2.77$ (s, 1H), 2.54 (ddd, $J=12.2$, 6.9, $2.5 \mathrm{~Hz}, 1 \mathrm{H}), 2.17$ (ddd, $J=16.1,6.0,2.8 \mathrm{~Hz}, 1 \mathrm{H}), 2.14-2.07(\mathrm{~m}, 2 \mathrm{H}), 2.01(\mathrm{~s}, 3 \mathrm{H})$, $1.89-1.82(\mathrm{~m}, 3 \mathrm{H}), 1.78-1.68(\mathrm{~m}, 2 \mathrm{H}), 1.77(\mathrm{dd}, J=14.0,11.8 \mathrm{~Hz}, 1 \mathrm{H}), 1.64-1.56$ $(\mathrm{m}, 3 \mathrm{H}), 1.52-1.43(\mathrm{~m}, 4 \mathrm{H}), 1.41(\mathrm{dd}, J=13.1,4.4 \mathrm{~Hz}, 1 \mathrm{H}), 1.36-1.31(\mathrm{~m}, 2 \mathrm{H}), 1.02$ (d, $J=6.6 \mathrm{~Hz}, 3 \mathrm{H}), 0.94$ (s, 3H), 0.91 (d, $J=6.9 \mathrm{~Hz}, 3 \mathrm{H}), 0.83$ (d, $J=7.0 \mathrm{~Hz}, 3 \mathrm{H}), 0.82$ (d, $J=6.9 \mathrm{~Hz}, 3 \mathrm{H}), 0.59(\mathrm{~s}, 3 \mathrm{H})$.

${ }^{13} \mathrm{C}-N M R: \quad\left(126 \mathrm{MHz}, \mathrm{CDCl}_{3}\right)$; $\delta[\mathrm{ppm}]=198.4,171.0,165.4,135.2,132.6,119.8,77.4$ (ov), 70.8, $56.2,55.9,44.8,43.8,43.0,40.6,40.5,39.0,33.2,32.7,30.2,28.0,26.5,22.6,22.0$, $21.5,21.3,20.1,19.8,17.7,16.3,12.8$.

IR: $\quad$ (neat); $\tilde{v}\left[\mathrm{~cm}^{-1}\right]=3414(\mathrm{br} \mathrm{w}), 2955(\mathrm{~s}), 2871(\mathrm{~m}), 1718(\mathrm{~m}), 1679(\mathrm{~s}), 1457(\mathrm{w}), 1382$ (m), 1365 (m), 1267 (s), 1245 (s), 11259 (w), $1118(w), 1103$ (w), 1045 (w), $1019(w)$, $974(w), 871(w), 756(m)$.

HRMS: $\quad$ (ESI-TOF); $m / z$ calcd. for $\mathrm{C}_{30} \mathrm{H}_{46} \mathrm{O}_{4} \mathrm{Na}^{+}[\mathrm{M}+\mathrm{Na}]^{+}:$493.3288, found: 493.3279. 
Opt. act. $\quad[a]_{D}^{25}=+2.1\left(c=1.00, \mathrm{CHCl}_{3}\right)$.

All characterization data were consistent with those reported in the literature (IR and ${ }^{1} \mathrm{H}-\mathrm{NMR},{ }^{[4]}{ }^{13} \mathrm{C}-\mathrm{NMR},{ }^{[6]}$ a and m.p[7]).

(22E)-5,6-Epoxy-6-oxo-5,6-secoergosta-4,7,22-trien-3ß-yl acetate (11)
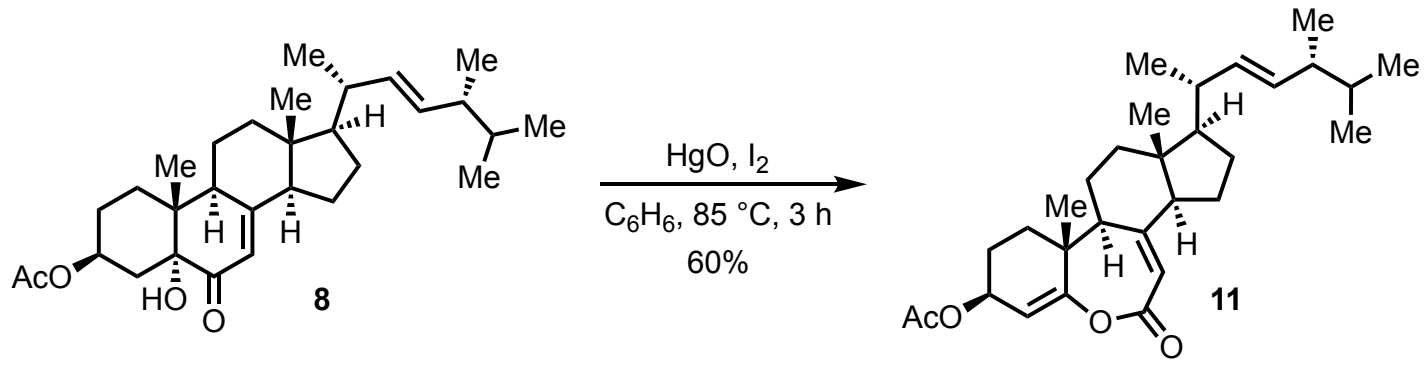

Through a solution of Burawoy ketone (8) $(50.0 \mathrm{mg}, 106 \mu \mathrm{mol}, 1.0$ eq.) in benzene $(5 \mathrm{~mL})$ was bubbled argon via cannula for $10 \mathrm{~min}$. lodine $(65.0 \mathrm{mg}, 255 \mu \mathrm{mol}, 2.4 \mathrm{eq}$ ) and $\mathrm{HgO}$ (yellow, $62.0 \mathrm{mg}, 287 \mu \mathrm{mol}$, 2.7 eq.) were added and the resulting mixture was heated to $85^{\circ} \mathrm{C}$ for $3 \mathrm{~h}$. The reaction mixture was cooled to $25^{\circ} \mathrm{C}$, filtered through a plug of Celite ${ }^{\circledR}$, and rinsed with EtOAc $(15 \mathrm{~mL})$. The organic phase was washed sequentially with $\mathrm{Na}_{2} \mathrm{~S}_{2} \mathrm{O}_{3}$ (sat. aq., $25 \mathrm{~mL}$ ) and brine (sat., $25 \mathrm{~mL}$ ), dried over $\mathrm{MgSO}_{4}$, and the solvent was removed under reduced pressure. Column chromatography (silica gel, nhexane/EtOAc 5:1) gave enol ester $11(29.9 \mathrm{mg}, 63.8 \mu \mathrm{mol}, 60 \%)$ as a colorless oil.

TLC: $\quad R_{\mathrm{f}}=0.37($ nhexane/EtOAc 5:1).

1H-NMR: $\quad\left(600 \mathrm{MHz}, \mathrm{CDCl}_{3}\right) ; \delta[\mathrm{ppm}]=5.64(\mathrm{~s}, 1 \mathrm{H}), 5.47(\mathrm{~d}, J=4.5 \mathrm{~Hz}, 1 \mathrm{H}), 5.30(\mathrm{dt}, J=4.7,2.3$ $\mathrm{Hz}, 1 \mathrm{H}$ ), 5.24 (dd, $J=15.3,7.8 \mathrm{~Hz}, 1 \mathrm{H}), 5.14$ (dd, $J=15.3,7.7 \mathrm{~Hz}, 1 \mathrm{H}$ ), 2.30 (ddd, $J=$ 12.6, 4.7, $2.1 \mathrm{~Hz}, 1 \mathrm{H}), 2.12(\mathrm{dd}, J=12.0,6.5 \mathrm{~Hz}, 1 \mathrm{H}), 2.06-2.01(\mathrm{~m}, 5 \mathrm{H}), 1.98-1.90(\mathrm{~m}$, $1 \mathrm{H}), 1.88-1.83(\mathrm{~m}, 1 \mathrm{H}), 1.82-1.73(\mathrm{~m}, 4 \mathrm{H}), 1.72-1.66(\mathrm{~m}, 1 \mathrm{H}), 1.61-1.54(\mathrm{~m}, 2 \mathrm{H})$, $1.50-1.43(\mathrm{~m}, 2 \mathrm{H}), 1.42-1.35(\mathrm{~m}, 3 \mathrm{H}), 1.12(\mathrm{~s}, 3 \mathrm{H}), 1.01(\mathrm{~d}, J=6.6 \mathrm{~Hz}, 3 \mathrm{H}), 0.91(\mathrm{~d}, J$ $=6.8 \mathrm{~Hz}, 3 \mathrm{H}), 0.83(\mathrm{~d}, J=6.8 \mathrm{~Hz}, 3 \mathrm{H}), 0.81(\mathrm{~d}, J=6.8 \mathrm{~Hz}, 3 \mathrm{H}), 0.61(\mathrm{~s}, 3 \mathrm{H})$.

${ }^{13} \mathrm{C}-\mathrm{NMR}: \quad\left(151 \mathrm{MHz}, \mathrm{CDCl}_{3}\right) ; \delta[\mathrm{ppm}]=170.6,165.1,159.2,158.3,135.0,132.8,113.2,110.0,66.6$, $58.4,56.5,48.5,47.2,43.0,40.4,39.6,38.9,33.2$, 30.9, 27.9, 25.4, 24.5, 22.7, 21.4, 21.2, 20.3, 20.1, 19.8, 17.7, 12.6.

IR: $\quad\left(\right.$ neat); $\tilde{v}\left[\mathrm{~cm}^{-1}\right]=2954(\mathrm{~m}), 2925(\mathrm{~m}), 2871(\mathrm{w}), 1722(\mathrm{~s}), 1668(\mathrm{~m}), 1456(\mathrm{w}), 1371(\mathrm{w})$, $1233(\mathrm{~s}), 1138(\mathrm{~m}), 1012(\mathrm{w}), 996(\mathrm{w}), 960(\mathrm{~m}), 891(\mathrm{w}), 865(\mathrm{w})$.

HRMS: (ESI-TOF); $\mathrm{m} / \mathrm{z}$ calcd. for $\mathrm{C}_{30} \mathrm{H}_{44} \mathrm{O}_{4} \mathrm{Na}^{+}[\mathrm{M}+\mathrm{Na}]^{+}: 491.3132$, found: 491.3124.

Opt. act. $[\mathrm{a}]_{\mathrm{D}}^{22}=+42.4\left(c=1.00, \mathrm{CHCl}_{3}\right)$. 


\subsection{Synthesis of Herbarulide (2)}

(22E)-5a-Hydroxyergosta-7,22-diene-3,6-dione (7)
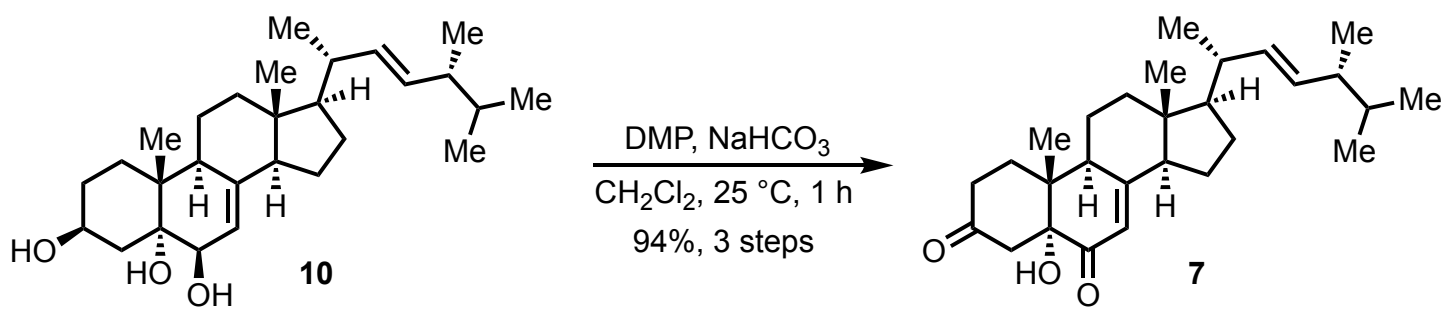

To a stirred solution of triol 10 (71 mg, $0.16 \mathrm{mmol}, 1.0$ eq.) in $\mathrm{CH}_{2} \mathrm{Cl}_{2}(1.6 \mathrm{~mL})$ at $25^{\circ} \mathrm{C}$ were added $\mathrm{NaHCO}_{3}$ (81 mg, $0.96 \mathrm{mmol}, 6.0$ eq.) and Dess-Martin periodinane (136 mg, $320 \mu \mathrm{mol}, 2.0$ eq.), and the reaction mixture was stirred at this temperature for $1 \mathrm{~h}$. After diluting with $\mathrm{CH}_{2} \mathrm{Cl}_{2}(5 \mathrm{~mL}), \mathrm{Na}_{2} \mathrm{~S}_{2} \mathrm{O}_{3}$ (sat. aq., $5 \mathrm{~mL}$ ) was added and the mixture was vigorously stirred for $30 \mathrm{~min}$. The phases were separated, and the aqueous phase was extracted with $\mathrm{CH}_{2} \mathrm{Cl}_{2}(3 \times 10 \mathrm{~mL})$. The combined organic phases were sequentially washed with $\mathrm{NaHCO}_{3}$ (sat. aq., $25 \mathrm{~mL}$ ) and brine (sat., $25 \mathrm{~mL}$ ), dried over $\mathrm{MgSO}_{4}$, and the solvent was removed under reduced pressure. The residue was adsorbed on silica gel and column chromatography (silica gel, nhexane/EtOAc, 3:1) gave dione 7 (65 mg, $0.15 \mathrm{mmol}, 94 \%$, 3 steps from ergosterol) as a colorless solid.

M.p.: $\quad 230-232^{\circ} \mathrm{C}(\mathrm{EtOAC})$.

TLC: $\quad R_{\mathrm{f}}=0.31$ (nhexane/EtOAc 2:1).

1H-NMR: $\quad\left(500 \mathrm{MHz}\right.$, pyridine- $\left.\mathrm{d}_{5}\right) ; \delta[\mathrm{ppm}]=8.12(\mathrm{~s}, 1 \mathrm{H}), 5.98(\mathrm{t}, J=2.3 \mathrm{~Hz}, 1 \mathrm{H}), 5.29(\mathrm{dd}, J=$ 15.3, $7.7 \mathrm{~Hz}, 1 \mathrm{H}$ ), 5.20 (dd, $J=15.3,8.2 \mathrm{~Hz}, 1 \mathrm{H}), 3.25-3.16(\mathrm{~m}, 2 \mathrm{H}), 2.98$ (ddd, $J=$ 12.2, 7.0, $2.5 \mathrm{~Hz}, 1 \mathrm{H}), 2.61-2.52(\mathrm{~m}, 1 \mathrm{H}), 2.52-2.41(\mathrm{~m}, 2 \mathrm{H}), 2.10-2.00(\mathrm{~m}, 3 \mathrm{H})$, $1.94-1.85(\mathrm{~m}, 2 \mathrm{H}), 1.77-1.69(\mathrm{~m}, 2 \mathrm{H}), 1.69-1.62(\mathrm{~m}, 1 \mathrm{H}), 1.57-1.45(\mathrm{~m}, 3 \mathrm{H}), 1.40$ - $1.32(\mathrm{~m}, 1 \mathrm{H}), 1.32-1.25(\mathrm{~m}, 2 \mathrm{H}), 1.24(\mathrm{~s}, 3 \mathrm{H}), 1.08(\mathrm{~d}, J=6.6 \mathrm{~Hz}, 3 \mathrm{H}), 0.99$ (d, $J=$ $6.8 \mathrm{~Hz}, 3 \mathrm{H}), 0.90(\mathrm{~d}, J=4.3 \mathrm{~Hz}, 3 \mathrm{H}), 0.89(\mathrm{~d}, J=4.2 \mathrm{~Hz}, 3 \mathrm{H}), 0.64(\mathrm{~s}, 3 \mathrm{H})$.

13C-NMR: $\quad\left(126 \mathrm{MHz}\right.$, pyridine- $\left.\mathrm{d}_{5}\right) ; \delta[\mathrm{ppm}]=210.5,198.9,165.2,135$ (ov), 132.9, 120.7, 80.2, $56.5,56.3,45.7,45.1,44.6,43.5,42.0,41.1,39.5,38.4,33.8,32.9,28.7,23.2,22.7$, 21.8, 20.6, 20.3, 18.3, 16.0, 13.2 .

IR: $\quad\left(\right.$ neat); $\tilde{v}\left[\mathrm{~cm}^{-1}\right]=2953(\mathrm{~m}), 2922(\mathrm{~s}), 2853(\mathrm{~m}), 1740(\mathrm{w}), 1460(\mathrm{w}), 1377(\mathrm{w}), 1217(\mathrm{w})$. HRMS: (ESI-TOF); $m / z$ calcd. for $\mathrm{C}_{28} \mathrm{H}_{42} \mathrm{O}_{3} \mathrm{Na}^{+}[\mathrm{M}+\mathrm{Na}]+:$ 449.3026, found: 449.3031.

Opt. act. $[\mathrm{a}]_{\mathrm{D}}^{22}=+34.8\left(c=1.00, \mathrm{CHCl}_{3}\right)$.

All characterization data were consistent with those reported in the literature (m.p., IR and ${ }^{1} \mathrm{H}-\mathrm{NMR}$ ). ${ }^{[8]}$ 

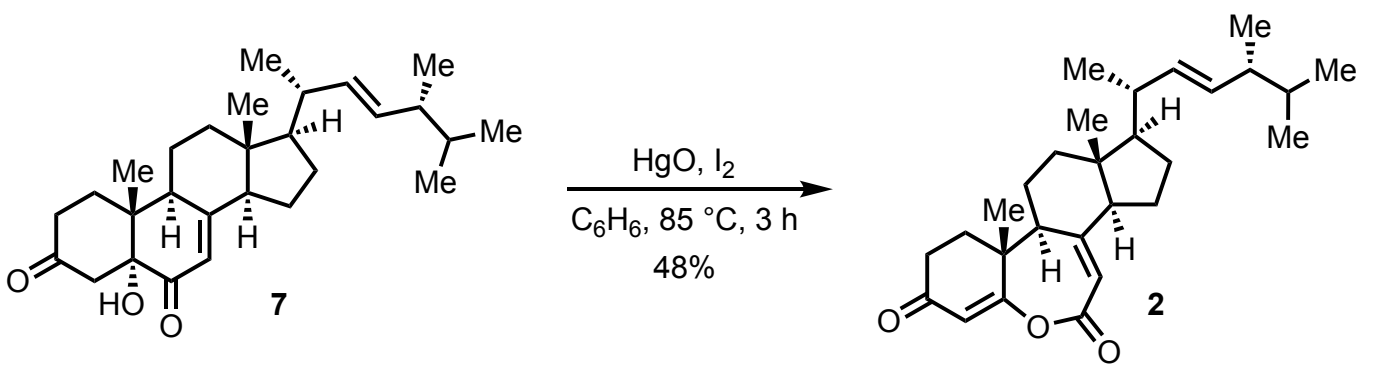

Through a solution of dione 7 (9.8 mg, $23 \mu \mathrm{mol}, 1.0$ eq.) in benzene $(2.5 \mathrm{~mL})$ was bubbled argon via cannula for $10 \mathrm{~min}$. lodine (14 mg, $55 \mu \mathrm{mol}, 2.4$ eq.) and $\mathrm{HgO}$ (yellow, $13 \mathrm{mg}, 62 \mu \mathrm{mol}, 2.7$ eq.) were added and the resulting mixture was heated to $85^{\circ} \mathrm{C}$ for $3 \mathrm{~h}$. The reaction mixture was cooled to $25^{\circ} \mathrm{C}$, filtered through a plug of Celite ${ }^{\circledR}$, and rinsed with EtOAc $(10 \mathrm{~mL})$. The organic phase was washed sequentially with $\mathrm{Na}_{2} \mathrm{~S}_{2} \mathrm{O}_{3}$ (sat. aq., $20 \mathrm{~mL}$ ) and brine (sat., $20 \mathrm{~mL}$ ), dried over $\mathrm{MgSO}_{4}$, and the solvent was removed under reduced pressure. Column chromatography (silica gel, nhexane/EtOAc 5:1 $\rightarrow$ 3:1) gave herbarulide (2) (4.7 mg, $11 \mu \mathrm{mol}, 48 \%)$ as a colorless solid.

M.p.: $\quad$ Decomposition at $200^{\circ} \mathrm{C}(\mathrm{EtOAC})$.

TLC: $\quad R_{\mathfrak{f}}=0.24$ (nhexane/EtOAc 3:1).

1H-NMR: $\quad\left(600 \mathrm{MHz}, \mathrm{CDCl}_{3}\right) ; \delta[\mathrm{ppm}]=5.75(\mathrm{~s}, 1 \mathrm{H}), 5.72(\mathrm{t}, J=1.8 \mathrm{~Hz}, 1 \mathrm{H}), 5.25(\mathrm{dd}, J=15.3,7.8$ $\mathrm{Hz}, 1 \mathrm{H}$ ), 5.15 (dd, $J=15.3,8.5 \mathrm{~Hz}, 1 \mathrm{H}), 2.53$ (ddd, $J=12.5,4.8,2.1 \mathrm{~Hz}, 1 \mathrm{H}$ ), 2.48 (dd, $J$ $=14.0,4.8 \mathrm{~Hz}, 1 \mathrm{H}), 2.46-2.40(\mathrm{~m}, 1 \mathrm{H}), 2.19(\mathrm{ddd}, J=14.1,4.9,2.2 \mathrm{~Hz}, 1 \mathrm{H}), 2.14$ (ddd, $J=11.8,6.7,1.3 \mathrm{~Hz}, 1 \mathrm{H}), 2.11(\mathrm{ddd}, J=13.1,4.1,2.8 \mathrm{~Hz}, 1 \mathrm{H}), 2.07-2.02(\mathrm{~m}, 1 \mathrm{H}), 1.98$ (td, $J=14.2,5.4 \mathrm{~Hz}, 1 \mathrm{H}), 1.88-1.82(\mathrm{~m}, 2 \mathrm{H}), 1.81-1.75(\mathrm{~m}, 1 \mathrm{H}), 1.69$ (qd, $J=13.3,4.1$ $\mathrm{Hz}, 1 \mathrm{H}), 1.61-1.56(\mathrm{~m}, 1 \mathrm{H}), 1.52-1.45(\mathrm{~m}, 3 \mathrm{H}), 1.42-1.35(\mathrm{~m}, 2 \mathrm{H}), 1.24(\mathrm{~s}, 3 \mathrm{H}), 1.02$ $(\mathrm{d}, J=6.6 \mathrm{~Hz}, 3 \mathrm{H}), 0.92(\mathrm{~d}, J=6.8 \mathrm{~Hz}, 3 \mathrm{H}), 0.84(\mathrm{~d}, J=6.8 \mathrm{~Hz}, 3 \mathrm{H}), 0.82(\mathrm{~d}, J=6.8 \mathrm{~Hz}$, $3 \mathrm{H}), 0.64(\mathrm{~s}, 3 \mathrm{H})$.

13C-NMR: $\quad\left(151 \mathrm{MHz}, \mathrm{CDCl}_{3}\right) ; \delta[\mathrm{ppm}]=198.6,174.0,162.6,159.6,134.9,133.0,114.8,113.5,58.3$, $56.5,47.4,47.1,43.0,40.5,40.4,39.3,34.1,33.3,33.2,27.8,25.5,22.7,21.2,20.1$ (2C), $19.8,17.7,12.6$.

IR: $\quad\left(\right.$ neat); $\tilde{v}\left[\mathrm{~cm}^{-1}\right]=2955(\mathrm{~s}), 2871(\mathrm{~m}), 1733(\mathrm{~s}), 1673(\mathrm{~s}), 1622(\mathrm{~s}), 1457(\mathrm{~m}), 1340(\mathrm{~m})$, $1186(\mathrm{~m}), 1138(\mathrm{~s}), 864(\mathrm{w}), 764(\mathrm{~m})$.

HRMS: (ESI-TOF); $\mathrm{m} / \mathrm{z}$ calcd. for $\mathrm{C}_{28} \mathrm{H}_{40} \mathrm{O}_{3} \mathrm{Na}^{+}[\mathrm{M}+\mathrm{Na}]^{+}:$447.2870, found: 447.2854.

Opt. act. $\quad[\mathrm{a}]_{\mathrm{D}}^{22}=+176.4\left(c=0.18, \mathrm{CH}_{2} \mathrm{Cl}_{2}\right)$, lit.: $[\mathrm{a}]_{\mathrm{D}}^{22}=+55\left(c=0.185, \mathrm{CH}_{2} \mathrm{Cl}_{2}\right) \cdot{ }^{[9]}$

$$
\begin{aligned}
& {[\mathrm{a}]_{\mathrm{D}}^{22}=+162.9\left(c=0.50, \mathrm{CH}_{2} \mathrm{Cl}_{2}\right) .} \\
& {[\mathrm{a}]_{\mathrm{D}}^{22}=+186.4\left(c=0.18, \mathrm{CHCl}_{3}\right) .}
\end{aligned}
$$




$$
[\mathrm{a}]_{\mathrm{D}}^{22}=+172.3\left(c=0.50, \mathrm{CHCl}_{3}\right) .
$$

All characterization data except for the optical rotation were consistent with those reported in the literature.[9]

\subsection{Synthesis of (22E)-5,6-Epoxy-5,6-secocampesta-4,7,22-triene-3,6- dione (1)}

5a-Hydroxy-3,6-dioxopregn-7-ene-20a-carboxaldehyde (S3)

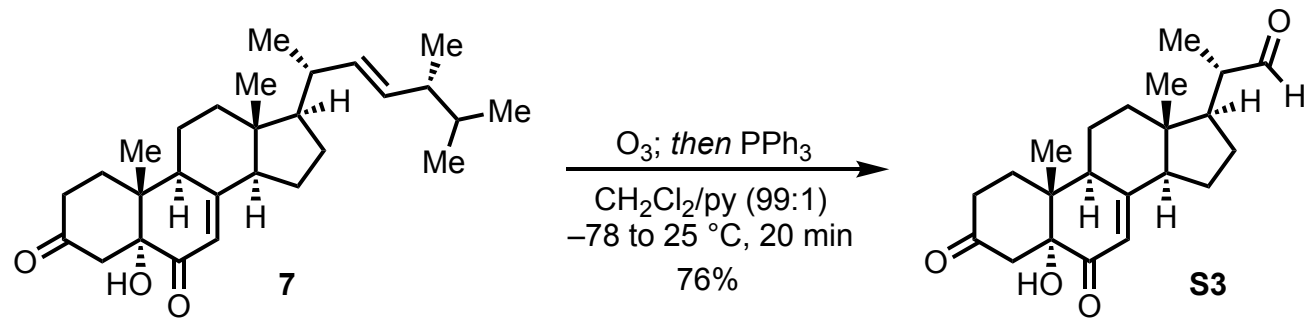

A stream of ozone-rich oxygen was passed through a solution of dione 7 (100 mg, $234 \mu \mathrm{mol}, 1.0 \mathrm{eq}$.) in $\mathrm{CH}_{2} \mathrm{Cl}_{2} /$ pyridine $\left(99: 1,10 \mathrm{~mL}\right.$ ) at $-78{ }^{\circ} \mathrm{C}$ for $20 \mathrm{~min}$. $\mathrm{PPh}_{3}$ (122 mg, $468 \mu \mathrm{mol}, 2.0$ eq.) was added and the reaction mixture was allowed to warm to $25^{\circ} \mathrm{C}$ over $16 \mathrm{~h}$. The solvent was removed, the residue was adsorbed on silica gel and column chromatography (silica gel, nhexane/EtOAc 2:1 $\rightarrow$ 1:2) gave aldehyde S3 (63.9 mg, $178 \mu \mathrm{mol}, 76 \%$ ) as a crystalline solid.

M.p.: $\quad 225-227^{\circ} \mathrm{C}\left(\mathrm{CHCl}_{3}\right)$.

TLC: $\quad R_{\mathrm{f}}=0.35($ nhexane/EtOAc $1: 1)$.

1H-NMR: $\quad\left(500 \mathrm{MHz}, \mathrm{CDCl}_{3}\right) ; \delta[\mathrm{ppm}]=9.59(\mathrm{~d}, J=3.0 \mathrm{~Hz}, 1 \mathrm{H}), 5.70(\mathrm{t}, J=2.3 \mathrm{~Hz}, 1 \mathrm{H}), 2.93(\mathrm{~s}, 1 \mathrm{H})$, $2.77(\mathrm{~d}, J=16.1 \mathrm{~Hz}, 1 \mathrm{H}), 2.69$ (ddd, $J=12.2,6.9,2.6 \mathrm{~Hz}, 1 \mathrm{H}), 2.54(\mathrm{dd}, J=16.1,1.9 \mathrm{~Hz}$, $1 \mathrm{H}), 2.44-2.35(\mathrm{~m}, 3 \mathrm{H}), 2.24-2.17(\mathrm{~m}, 2 \mathrm{H}), 2.11$ (ddd, $J=12.7,4.5,2.7 \mathrm{~Hz}, 1 \mathrm{H}), 2.07-$ $1.98(\mathrm{~m}, 1 \mathrm{H}), 1.90(\mathrm{ddd}, J=13.1,6.0,2.7 \mathrm{~Hz}, 1 \mathrm{H}), 1.85-1.78(\mathrm{~m}, 1 \mathrm{H}), 1.79-1.73(\mathrm{~m}$, 2H), $1.72-1.67(\mathrm{~m}, 1 \mathrm{H}), 1.63-1.57(\mathrm{~m}, 1 \mathrm{H}), 1.54(\mathrm{dd}, J=13.0,4.5 \mathrm{~Hz}, 1 \mathrm{H}), 1.52-1.44$ $(\mathrm{m}, 1 \mathrm{H}), 1.16(\mathrm{~d}, J=6.9 \mathrm{~Hz}, 3 \mathrm{H}), 1.13(\mathrm{~s}, 3 \mathrm{H}), 0.66(\mathrm{~s}, 3 \mathrm{H})$.

13C-NMR: $\quad\left(126 \mathrm{MHz}, \mathrm{CDCl}_{3}\right) ; \delta[\mathrm{ppm}]=210.3,204.4,197.3,164.8,120.0,79.8,55.1,51.1,49.6$, 45.2, 44.6, 43.8, 41.0, 38.7, 37.4, 32.0, 26.7, 23.0, 22.0, 15.9, 13.7, 13.0.

IR: $\quad\left(\right.$ neat); $\tilde{v}\left[\mathrm{~cm}^{-1}\right]=3349(\mathrm{br} \mathrm{w}), 2954(\mathrm{w}), 2922(\mathrm{~m}), 2874(\mathrm{w}), 2850(\mathrm{w}), 1712(\mathrm{~m}), 1671(\mathrm{~s})$, $1620(w), 1458(w), 1374(w), 1232(w), 1158(w), 1124(w), 873(w) 758(w)$.

HRMS: (ESI-TOF); $m / z$ calcd. for $\mathrm{C}_{22} \mathrm{H}_{30} \mathrm{O}_{4} \mathrm{Na}^{+}[\mathrm{M}+\mathrm{Na}]+:$ 381.2036, found: 381.2031.

Opt. act. $[\mathrm{a}]_{\mathrm{D}}^{22}=+48.5\left(c=1.00, \mathrm{CHCl}_{3}\right)$. 

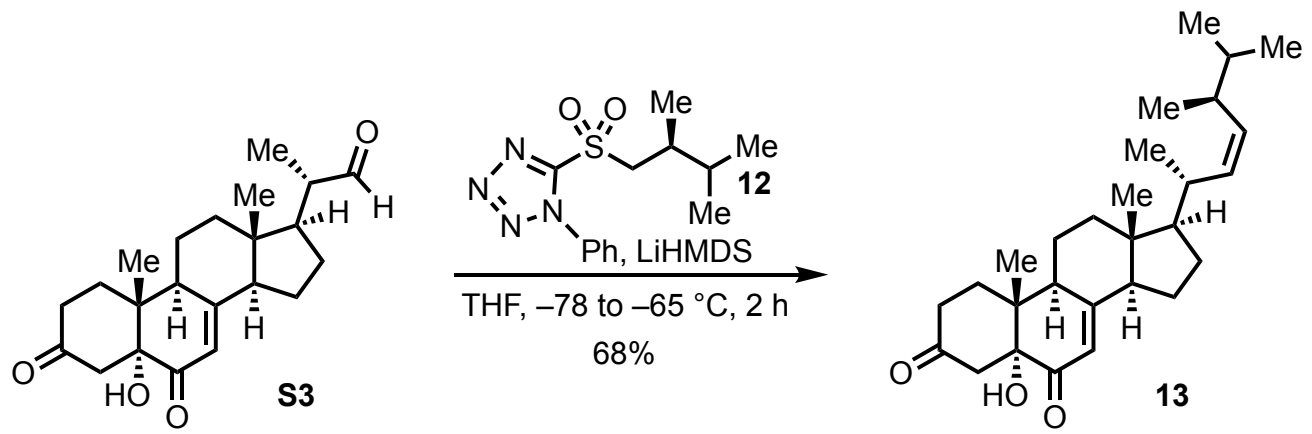

To a solution of hexamethyldisilazane $\left(119 \mu \mathrm{L}, 578 \mu \mathrm{mol}, 3.26\right.$ eq.) in THF $(0.2 \mathrm{~mL})$ at $0{ }^{\circ} \mathrm{C}$ was added $n B u L i(1.6 \mathrm{~m}$ in $n$ hexane, $343 \mu \mathrm{L}, 549 \mu \mathrm{mol}, 3.1$ eq.) and it was stirred for $15 \mathrm{~min}$ at this temperature before the solution was added to sulfone 12 ( $261 \mathrm{mg}, 885 \mu \mathrm{mol}, 5.0$ eq.) in THF (2.5 mL) at $-78{ }^{\circ} \mathrm{C}$. The resulting bright yellow solution was stirred at $-65^{\circ} \mathrm{C}$ for $1 \mathrm{~h}$ when a solution of aldehyde S3 $(63.5 \mathrm{mg}, 177 \mu \mathrm{mol}$, 1.0 eq.) in THF ( $2 \mathrm{~mL})$ was added to the reaction mixture over $30 \mathrm{~min}$. The reaction was stirred at $-65^{\circ} \mathrm{C}$ for further $30 \mathrm{~min}$ before EtOAc $(3 \mathrm{~mL})$ and $\mathrm{HCl}\left(1 \mathrm{M}\right.$ in $\left.\mathrm{H}_{2} \mathrm{O}, 5 \mathrm{~mL}\right)$ were added to the reaction mixture. The aqueous phase was extracted with EtOAc $(3 \times 10 \mathrm{~mL})$ and the combined organic phases were washed sequentially with $\mathrm{NaHCO}_{3}$ (sat. aq., $20 \mathrm{~mL}$ ) and brine (sat., $20 \mathrm{~mL}$ ), dried over $\mathrm{MgSO}_{4}$, and the solvent was removed under reduced pressure. Column chromatography (silica gel, nhexane/EtOAc 12:1 $\rightarrow$ 5:1 $\rightarrow$ 2:1) gave (22Z)-olefin 13 (51 mg, $0.12 \mathrm{mmol}, 68 \%, 52 \%$ over two steps) as a crystalline solid and reisolated sulfone 12 (193 mg, $655 \mu \mathrm{mol})$ as a colorless oil.

The synthesis of sulfone 12 was carried out starting from $(R)$-Roche ester as previously described by us. ${ }^{[10]}$

M.p.: $\quad 245-248^{\circ} \mathrm{C}(\mathrm{EtOAc})$.

TLC: $\quad R_{\mathrm{f}}=0.31$ (nhexane/EtOAc 2:1).

1H-NMR: $\quad\left(600 \mathrm{MHz}\right.$, pyridine- $\left.\mathrm{d}_{5}\right) ; \delta[\mathrm{ppm}]=8.10(\mathrm{~s}, 1 \mathrm{H}), 5.98(\mathrm{t}, J=2.3 \mathrm{~Hz}, 1 \mathrm{H}), 5.16(\mathrm{t}, J=10.5$ $\mathrm{Hz}, 1 \mathrm{H}), 5.10(\mathrm{t}, J=10.5 \mathrm{~Hz}, 1 \mathrm{H}), 3.27-3.14(\mathrm{~m}, 2 \mathrm{H}), 3.03-2.93(\mathrm{~m}, 1 \mathrm{H}), 2.60-2.42$ $(\mathrm{m}, 4 \mathrm{H}), 2.34-2.25(\mathrm{~m}, 1 \mathrm{H}), 2.13-2.01(\mathrm{~m}, 2 \mathrm{H}), 1.93-1.86(\mathrm{~m}, 1 \mathrm{H}), 1.81-1.73(\mathrm{~m}$, 2H), 1.68 (qd, $J=13.1,4.4 \mathrm{~Hz}, 1 \mathrm{H}), 1.56-1.48(\mathrm{~m}, 2 \mathrm{H}), 1.46-1.35(\mathrm{~m}, 2 \mathrm{H}), 1.35-1.26$ $(\mathrm{m}, 2 \mathrm{H}), 1.23(\mathrm{~s}, 3 \mathrm{H}), 1.08(\mathrm{~d}, J=5.8 \mathrm{~Hz}, 3 \mathrm{H}), 0.97(\mathrm{~d}, J=6.7 \mathrm{~Hz}, 3 \mathrm{H}), 0.92-0.86(\mathrm{~m}$, $6 \mathrm{H}), 0.72(\mathrm{~d}, J=3.7 \mathrm{~Hz}, 3 \mathrm{H})$.

${ }^{13}$ C-NMR: $\quad\left(151 \mathrm{MHz}\right.$, pyridine- $\left.\mathrm{d}_{5}\right) ; \delta[\mathrm{ppm}]=210.5,198.9,165.2,135.6,132.2,120.7,80.2,56.7$, $56.3,45.7,45.1,44.7,42.0,39.5,39.0,38.3,35.4,34.0,32.9,28.6,23.1,22.7,21.3,21.0$, 20.4, 19.3, 16.0, 13.2. 
IR: $\quad\left(\right.$ neat); $\tilde{v}\left[\mathrm{~cm}^{-1}\right]=2954(\mathrm{~m}), 2023(\mathrm{~s}), 2853(\mathrm{~m}), 1739(\mathrm{~m}), 1459(\mathrm{w}), 1376(\mathrm{w}), 1217(\mathrm{w})$, $757(w)$.

HRMS: $\quad$ (ESI-TOF); $m / z$ calcd. for $\mathrm{C}_{28} \mathrm{H}_{42} \mathrm{O}_{3} \mathrm{Na}^{+}[\mathrm{M}+\mathrm{Na}]^{+}: 449.3026$, found: 449.3024 .

Opt. act. $[\mathrm{a}]_{\mathrm{D}}^{20}=+27.5\left(c=1.00, \mathrm{CHCl}_{3}\right)$.

22,23-Dibromo-5a-hydroxycampesta-7-ene-3,6-dione (S4)

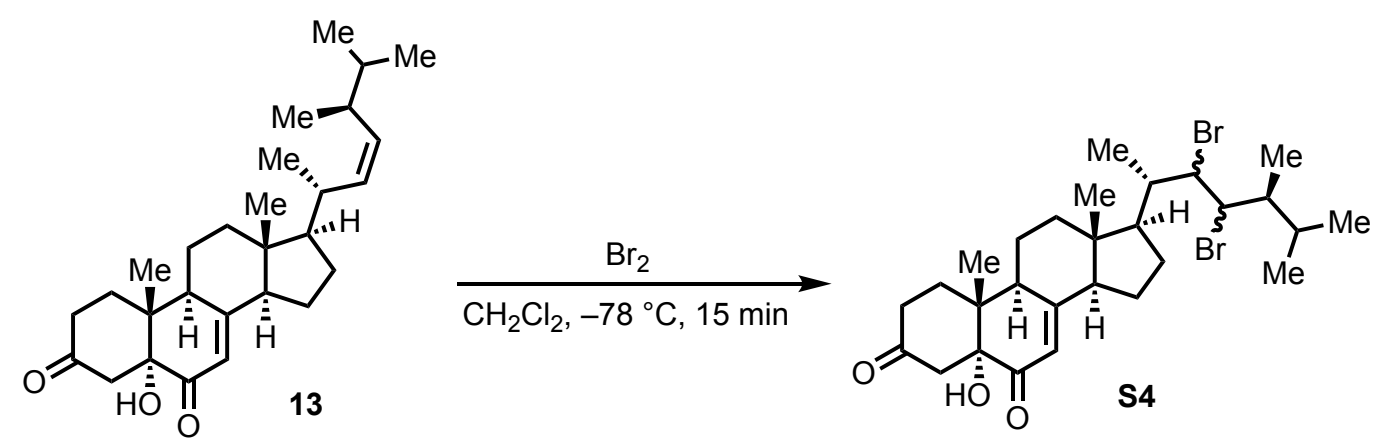

A stock solution of bromine (92 $\mu \mathrm{L}, 0.18 \mathrm{mmol}, 100$ eq.) in $\mathrm{CH}_{2} \mathrm{Cl}_{2}(5 \mathrm{~mL})$ was prepared by dissolution at $0{ }^{\circ} \mathrm{C}$. In a separate flask, a portion of this stock solution (50 $\mu \mathrm{L}$, equals $1.1 \mathrm{eq}$. bromine) was added to a solution of (22Z)-olefin 13 (7.8 mg, $18 \mu \mathrm{mol}, 1.0$ eq.) in $\mathrm{CH}_{2} \mathrm{Cl}_{2}(0.75 \mathrm{~mL})$ at $-78{ }^{\circ} \mathrm{C}$ and the reaction mixture was stirred at this temperature for $15 \mathrm{~min}$. The reaction was then diluted with $\mathrm{CH}_{2} \mathrm{Cl}_{2}(5 \mathrm{~mL})$ and quenched with $\mathrm{Na}_{2} \mathrm{~S}_{2} \mathrm{O}_{3}$ (sat. aq., $5 \mathrm{~mL}$ ). The aqueous phase was extracted with $\mathrm{CH}_{2} \mathrm{Cl}_{2}(3 \times 10 \mathrm{~mL})$ and the combined organic phases were washed with brine (sat., $25 \mathrm{~mL}$ ), dried over $\mathrm{MgSO}_{4}$, and the solvent was removed under reduced pressure. The yellow residue was used in the next step without further purification.

An analytically pure sample of dibromide diastereomers $\mathbf{S 4}$ (d.r. 2:1, determined by integration of $22-\mathrm{H}$ and 23- $\mathrm{H}$ signals $\delta[\mathrm{ppm}]=4.55$ (minor), 4.50 (major), 4.35 (major) and 3.97 (minor) in ${ }^{1} \mathrm{H}-\mathrm{NMR}$ ) could be obtained by column chromatography (silica gel, nhexane/EtOAc $3: 1 \rightarrow 2: 1$ ) as a light-yellow solid.

M.p.: $\quad 170-173^{\circ} \mathrm{C}\left(\mathrm{CH}_{2} \mathrm{Cl}_{2}\right)$.

TLC: $\quad R_{\mathrm{f}}=0.24$ (nhexane/EtOAc $2: 1$ ).

Significant signals of the minor diastereomer are marked with *.

1H-NMR: $\quad\left(600 \mathrm{MHz}, \mathrm{CDCl}_{3}\right) ; \delta[\mathrm{ppm}]=5.74-5.68(\mathrm{~m}, 1 \mathrm{H}), 4.55(\mathrm{dd}, J=6.2,1.5 \mathrm{~Hz}, 1 \mathrm{H})^{\star}, 4.50$ (dd, $J=9.8,2.1 \mathrm{~Hz}, 1 \mathrm{H}$ ), 4.35 (dd, $J=9.8,1.9 \mathrm{~Hz}, 1 \mathrm{H}$ ), $3.97(\mathrm{dd}, J=9.8,1.4 \mathrm{~Hz}, 1 \mathrm{H})^{\star}$, $2.82-2.77(\mathrm{~m}, 1 \mathrm{H}), 2.70-2.64(\mathrm{~m}, 1 \mathrm{H}), 2.56(\mathrm{dd}, J=16.2,1.8 \mathrm{~Hz}, 1 \mathrm{H}), 2.49(\mathrm{~s}, 1 \mathrm{H})$, $2.41-2.36(\mathrm{~m}, 2 \mathrm{H}), 2.27-2.22(\mathrm{~m}, 1 \mathrm{H}), 2.22-2.10(\mathrm{~m}, 2 \mathrm{H}), 2.09-2.02(\mathrm{~m}, 1 \mathrm{H}), 1.97$ (ddd, $J=9.8,6.9,2.6 \mathrm{~Hz}, 1 \mathrm{H})^{\star}, 1.94-1.88(\mathrm{~m}, 1 \mathrm{H}), 1.86-1.78(\mathrm{~m}, 3 \mathrm{H}), 1.78-1.73$ $(\mathrm{m}, 1 \mathrm{H}), 1.71-1.65(\mathrm{~m}, 2 \mathrm{H}), 1.62-1.54(\mathrm{~m}, 2 \mathrm{H}), 1.32(\mathrm{~d}, J=7.1 \mathrm{~Hz}, 3 \mathrm{H})^{\star}, 1.31-1.27$ $(\mathrm{m}, 1 \mathrm{H}), 1.23-1.17(\mathrm{~m}, 1 \mathrm{H}), 1.14(\mathrm{~s}, 3 \mathrm{H}), 1.14(\mathrm{~s}, 3 \mathrm{H})^{\star}, 1.01(\mathrm{~d}, J=6.3 \mathrm{~Hz}, 3 \mathrm{H}), 0.99$ 
$-0.96(\mathrm{~m}, 6 \mathrm{H}), 0.95(\mathrm{~d}, J=6.5 \mathrm{~Hz}, 3 \mathrm{H}), 0.86(\mathrm{~d}, J=6.9 \mathrm{~Hz}, 3 \mathrm{H})^{\star}, 0.75(\mathrm{~d}, J=6.8 \mathrm{~Hz}$, $3 \mathrm{H})^{\star}, 0.67(\mathrm{~s}, 3 \mathrm{H}), 0.66(\mathrm{~s}, 3 \mathrm{H})^{*}$.

13C-NMR: $\quad\left(151 \mathrm{MHz}, \mathrm{CDCl}_{3}\right) ; \delta[\mathrm{ppm}]=210.1,197.2,165.0,120.0^{*}, 119.9,79.9,68.9,66.9,62.1^{*}$, $60.4^{\star}, 55.5,55.5^{\star}, 55.1,53.1^{\star}, 45.6^{*}, 45.1^{\star}, 44.7,44.7^{\star}, 43.8,43.8,42.8,41.0,38.9$, $38.7^{*}, 38.7,37.5,32.8,32.1^{*}, 32.0,28.4^{*}, 26.9^{*}, 26.7,22.6^{\star}, 22.3,22.1,22.1^{\star}, 21.9^{*}$, $21.0,20.6,16.6^{*}, 16.0,15.9^{*}, 15.3^{*}, 14.0,13.3,13.3^{*}, 12.7,10.6^{*}$.

IR: $\quad\left(\right.$ neat); $\tilde{v}\left[\mathrm{~cm}^{-1}\right]=3342(\mathrm{br} \mathrm{w}), 2925(\mathrm{~m}), 2873(\mathrm{w}), 2857(\mathrm{w}), 1714(\mathrm{~m}), 1675(\mathrm{~s}), 1622$ $(w), 1459(w), 1380(w), 1232(w), 1159(w)$.

HRMS: (ESI-TOF); $m / z$ calcd. for $\mathrm{C}_{28} \mathrm{H}_{42} \mathrm{Br}_{2} \mathrm{O}_{3} \mathrm{Na}^{+}[\mathrm{M}+\mathrm{Na}]^{+}: 607.1393$, found: 607.1377.

Opt. act. $[\mathrm{a}]_{\mathrm{D}}^{22}=+16.6\left(c=1.00, \mathrm{CHCl}_{3}\right)$.

(22E)-5a-Hydroxycampesta-7,22-diene-3,6-dione (14) and (22Z)-5a-Hydroxycampesta-7,22-diene-3,6dione (13)
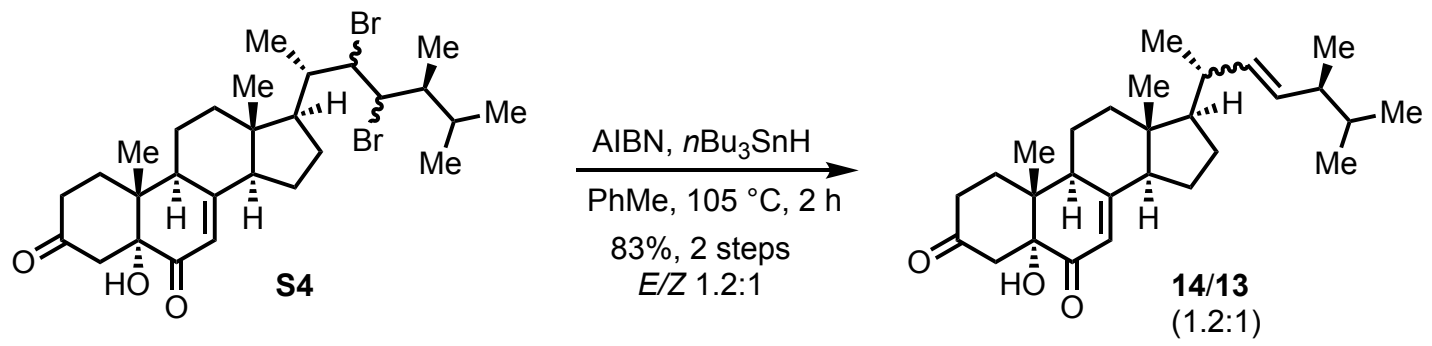

A solution of azobisisobutyronitrile ( $0.6 \mathrm{mg}, 3.6 \mu \mathrm{mol}, 0.2$ eq.), $n \mathrm{Bu} \mathrm{u}_{3} \mathrm{SnH}$ ( $24 \mu \mathrm{L}, 90 \mu \mathrm{mol}, 5.0$ eq.) and crude dibromide $\mathbf{S} 4$ (18 $\mu \mathrm{mol}, 1.0$ eq.) in toluene $(0.75 \mathrm{~mL})$ was degassed by applying three freeze-pump-thaw cycles before the reaction mixture was heated to $105^{\circ} \mathrm{C}$ for $2 \mathrm{~h}$. It was cooled to $25^{\circ} \mathrm{C}$ and the solvent was removed under reduced pressure. The residue was adsorbed on silica and column chromatography (silica gel, nhexane/EtOAc 3:1 $\rightarrow$ 2:1) gave a mixture of $(E)$-isomer 14 and (Z)-isomer 13 (E/Z 1.2:1, determined by integration of $22-\mathrm{H}$ and $23-\mathrm{H}$ signals $\delta[p p m]=5.22$ (major), 5.16 (major), 5.08 (minor) and 5.03 (minor) in ${ }^{1} \mathrm{H}-\mathrm{NMR}, 6.4 \mathrm{mg}, 15 \mu \mathrm{mol}, 83 \%$ over two steps) as a colorless solid.

M.p.: $\quad$ Decomposition at $238-240^{\circ} \mathrm{C}(\mathrm{EtOAC})$.

TLC: $\quad R_{\mathrm{f}}=0.28$ (nhexane/EtOAc $2: 1$ ).

Significant signals of the minor diastereomer are marked with *.

1H-NMR: $\quad\left(600 \mathrm{MHz}, \mathrm{CDCl}_{3}\right) ; \delta[\mathrm{ppm}]=5.71(\mathrm{t}, J=2.3 \mathrm{~Hz}, 1 \mathrm{H}), 5.22(\mathrm{dd}, J=15.2,7.9 \mathrm{~Hz}, 1 \mathrm{H})$, $5.16(\mathrm{dd}, J=15.2,8.3 \mathrm{~Hz}, 1 \mathrm{H}), 5.10-5.06(\mathrm{~m}, 1 \mathrm{H})^{\star}, 5.03(\mathrm{t}, J=10.5,1 \mathrm{H})^{\star}, 2.81(\mathrm{dd}$, $J=16.2,1.6 \mathrm{~Hz}, 1 \mathrm{H}), 2.68-2.62(\mathrm{~m}, 1 \mathrm{H}), 2.57(\mathrm{dd}, J=16.1,2.1 \mathrm{~Hz}, 1 \mathrm{H}), 2.45-2.36$ (m, 2H), $2.26(\mathrm{~s}, 1 \mathrm{H}), 2.22-2.13(\mathrm{~m}, 3 \mathrm{H}), 2.08-2.00(\mathrm{~m}, 1 \mathrm{H}), 1.95-1.89(\mathrm{~m}, 1 \mathrm{H})$, 
$1.87-1.77(\mathrm{~m}, 2 \mathrm{H}), 1.72(\mathrm{tt}, J=12.6,4.3 \mathrm{~Hz}, 1 \mathrm{H}), 1.68-1.63(\mathrm{~m}, 1 \mathrm{H}), 1.56-1.44(\mathrm{~m}$, $3 \mathrm{H}), 1.44-1.35(\mathrm{~m}, 2 \mathrm{H}), 1.33-1.28(\mathrm{~m}, 1 \mathrm{H}), 1.16(\mathrm{~s}, 3 \mathrm{H})^{*}, 1.15(\mathrm{~s}, 3 \mathrm{H}), 1.04(\mathrm{t}, J=$ $5.7 \mathrm{~Hz}, 3 \mathrm{H}), 0.99(\mathrm{~d}, J=6.5 \mathrm{~Hz}, 3 \mathrm{H})^{\star}, 0.92(\mathrm{~d}, J=6.8 \mathrm{~Hz}, 3 \mathrm{H})^{\star}, 0.92(\mathrm{~d}, J=6.8 \mathrm{~Hz}$, $3 \mathrm{H}), 0.88(\mathrm{~d}, J=6.9 \mathrm{~Hz}, 3 \mathrm{H}), 0.84(\mathrm{~d}, J=7.0 \mathrm{~Hz}, 3 \mathrm{H}), 0.83(\mathrm{~d}, J=6.9 \mathrm{~Hz}, 3 \mathrm{H})^{\star}, 0.67$ $(\mathrm{s}, 3 \mathrm{H})^{\star}, 0.64(\mathrm{~s}, 3 \mathrm{H})$.

13C-NMR: $\quad\left(151 \mathrm{MHz}, \mathrm{CDCl}_{3}\right) ; \delta[\mathrm{ppm}]=210.1,197.1,166.0,135.3,134.5^{\star}, 132.8,132.0^{\star}, 119.7$, $80.0,56.3^{*}, 56.1^{\star}, 56.0,44.9,44.8,43.9^{\star}, 43.9,43.2,41.0,41.0^{\star}, 40.5,39.0^{\star}, 38.9$, $38.5^{\star}, 37.5,34.7,33.5^{\star}, 33.3,32.1,28.3,27.9^{\star}, 22.7,22.6^{\star}, 22.2,21.3,20.8^{\star}, 20.5^{\star}$, $20.3,20.0^{\star}, 19.8,18.8^{\star}, 18.1,16.0^{\star}, 16.0,12.9^{\star}, 12.8$.

IR: $\quad\left(\right.$ neat); $\tilde{v}\left[\mathrm{~cm}^{-1}\right]=3334(\mathrm{br} \mathrm{w}), 2954(\mathrm{~m}), 2922(\mathrm{~s}), 2852(\mathrm{~m}), 1716(\mathrm{w}), 1674(\mathrm{~m}), 1622$ $(w), 1457(w), 1378(w), 1233(w), 874(w), 721(w)$.

HRMS: $\quad$ (ESI-TOF); $\mathrm{m} / z$ calcd. for $\mathrm{C}_{28} \mathrm{H}_{42} \mathrm{O}_{3} \mathrm{Na}^{+}[\mathrm{M}+\mathrm{Na}]+:$ 449.3026, found: 449.3032.

Opt. act. $[\mathrm{a}]_{\mathrm{D}}^{22}=+34.1\left(c=1.00, \mathrm{CHCl}_{3}\right)$.

(22E)-5,6-Epoxy-5,6-secocampesta-4,7,22-triene-3,6-dione (1) and (22Z)-5,6-Epoxy-5,6-secocampesta-4,7,22-triene-3,6-dione (22Z)-1
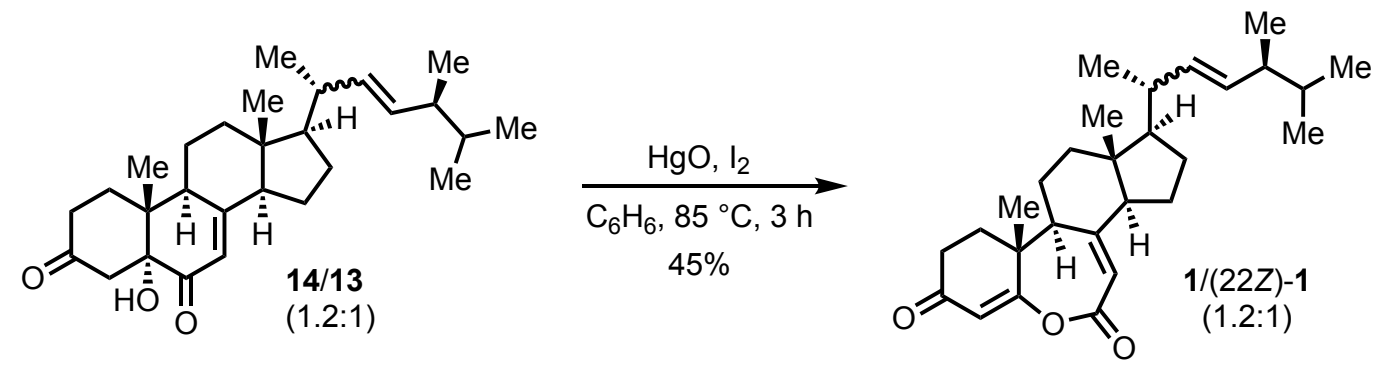

Through a mixture of $E$ and $Z$ dione 14 and 13 (9.9 mg, $23 \mu \mathrm{mol}, 1.0$ eq.) in benzene (2 $\mathrm{mL})$ was bubbled argon via cannula for $10 \mathrm{~min}$. lodine (14.0, $55.2 \mu \mathrm{mol}, 2.4$ eq.) and $\mathrm{HgO}$ (yellow, $13.5 \mathrm{mg}, 62.1 \mu \mathrm{mol}$, 2.7 eq.) were added and the resulting mixture was heated to $85^{\circ} \mathrm{C}$ for $3 \mathrm{~h}$. The reaction mixture was cooled to $25^{\circ} \mathrm{C}$, filtered through a plug of Celite ${ }^{\circledR}$, and rinsed with EtOAc $(10 \mathrm{~mL})$. The organic phase was washed sequentially with $\mathrm{Na}_{2} \mathrm{~S}_{2} \mathrm{O}_{3}$ (sat. aq., $250 \mathrm{~mL}$ ) and brine (sat., $250 \mathrm{~mL}$ ), dried over $\mathrm{MgSO}_{4}$, and the solvent was removed under reduced pressure. Column chromatography (silica gel, nhexane/EtOAc 5:1 $\rightarrow$ 3:1) gave a mixture of enol esters 1 and $22 Z-1$ (4.40 mg, $10.4 \mu \mathrm{mol}, 45 \%)$ as a colorless oil.

Separation of the diastereomers was performed using a pre-packed RP 18 column (Kinetex EVO, $5 \mu \mathrm{m}$, $250 \times 21 \mathrm{~mm}$ ), $\lambda=254 \mathrm{~nm}$, acetonitrile $/ \mathrm{H}_{2} \mathrm{O} 85: 15$, flow rate $=15 \mathrm{~mL} / \mathrm{min} ; \mathrm{t}_{\mathrm{R}}=20.40 \mathrm{~min}$ (major, 1), $18.25 \mathrm{~min}$ (minor, (22Z)-1) and gave 1 (2.4 mg, $5.6 \mu \mathrm{mol}, 20 \%$ over 3 steps) as a colorless oil and (22Z)-1 (2.0 mg, $4.7 \mu \mathrm{mol}, 17 \%$ over 3 steps) as a colorless solid. 


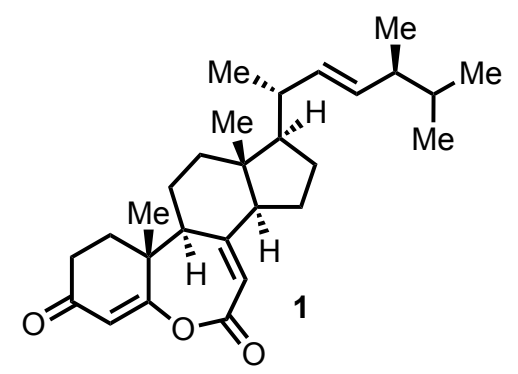

TLC: $\quad R_{\mathrm{f}}=0.28($ nhexane/EtOAc $3: 1)$.

1H-NMR: $\quad\left(600 \mathrm{MHz}, \mathrm{CDCl}_{3}\right) ; \delta[\mathrm{ppm}]=5.75(\mathrm{~s}, 1 \mathrm{H}), 5.72(\mathrm{~s}, 1 \mathrm{H}), 5.23(\mathrm{dd}, J=15.2,8.1 \mathrm{~Hz}, 1 \mathrm{H})$, $5.14(\mathrm{dd}, J=15.2,8.6 \mathrm{~Hz}, 1 \mathrm{H}), 2.56-2.51(\mathrm{~m}, 1 \mathrm{H}), 2.49$ (dd, $J=14.0,4.8 \mathrm{~Hz}, 1 \mathrm{H}), 2.43$ (ddd, $J=18.4,5.5,2.1 \mathrm{~Hz}, 1 \mathrm{H}$ ), 2.19 (ddd, $J=14.1,5.0,2.2 \mathrm{~Hz}, 1 \mathrm{H}$ ), 2.15 (dd, $J=11.9$, $6.6 \mathrm{~Hz}, 1 \mathrm{H}), 2.13-2.08(\mathrm{~m}, 1 \mathrm{H}), 2.07-2.01(\mathrm{~m}, 1 \mathrm{H}), 1.98(\mathrm{td}, J=14.3,5.6 \mathrm{~Hz}, 1 \mathrm{H}), 1.88$ $-1.82(\mathrm{~m}, 2 \mathrm{H}), 1.82-1.76(\mathrm{~m}, 1 \mathrm{H}), 1.69(\mathrm{qd}, J=13.3,4.1 \mathrm{~Hz}, 1 \mathrm{H}), 1.60-1.55(\mathrm{~m}, 1 \mathrm{H})$, $1.52-1.45(\mathrm{~m}, 3 \mathrm{H}), 1.42-1.35(\mathrm{~m}, 2 \mathrm{H}), 1.24(\mathrm{~s}, 3 \mathrm{H}), 1.02(\mathrm{~d}, J=6.6 \mathrm{~Hz}, 3 \mathrm{H}), 0.92(\mathrm{~d}, J$ $=6.8 \mathrm{~Hz}, 3 \mathrm{H}), 0.84(\mathrm{~d}, J=6.8 \mathrm{~Hz}, 3 \mathrm{H}), 0.82(\mathrm{~d}, J=6.7 \mathrm{~Hz}, 3 \mathrm{H}), 0.64(\mathrm{~s}, 3 \mathrm{H})$.

13C-NMR: $\quad\left(151 \mathrm{MHz}, \mathrm{CDCl}_{3}\right) ; \delta[\mathrm{ppm}]=198.6,174.0,162.6,159.6,135.1,133.1,114.8,113.5,58.3$, $56.4,47.4,47.1,43.2,40.5,40.5,39.3,34.1$, 33.3, 33.3, 28.1, 25.5, 22.8, 21.2, 20.3, 20.1, 19.8, 18.1, 12.6 .

IR: $\quad\left(\right.$ neat); $\tilde{v}\left[\mathrm{~cm}^{-1}\right]=2954(\mathrm{~s}), 2923(\mathrm{~s}), 2854(\mathrm{~m}), 1735(\mathrm{~s}), 1673(\mathrm{~m}), 1622(\mathrm{~m}), 1457(\mathrm{~m})$, 1377 (w), 1340 (w), $1280(w), 1232(\mathrm{~m}), 1186(\mathrm{~m}), 1138$ (s), $970(\mathrm{w}), 864(\mathrm{w})$.

HRMS: (ESI-TOF); $m / z$ calcd. for $\mathrm{C}_{28} \mathrm{H}_{40} \mathrm{O}_{3} \mathrm{Na}^{+}[\mathrm{M}+\mathrm{Na}]+:$ 447.2870, found: 447.2871.

Opt. act. $[a]_{D}^{22}=+160.1\left(c=0.22, \mathrm{CH}_{2} \mathrm{Cl}_{2}\right)$.

$$
[\mathrm{a}]_{\mathrm{D}}^{22}=+177.2\left(c=0.22, \mathrm{CHCl}_{3}\right) \text {. }
$$

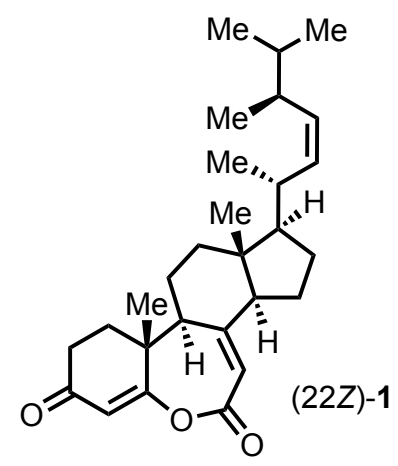

M.p.: $\quad 210-213^{\circ} \mathrm{C}\left(\mathrm{CHCl}_{3}\right)$.

TLC: $\quad R_{\mathrm{f}}=0.28($ nhexane/EtOAc $3: 1)$. 
1H-NMR: $\quad\left(600 \mathrm{MHz}, \mathrm{CDCl}_{3}\right) ; \delta[\mathrm{ppm}]=5.75(\mathrm{~s}, 1 \mathrm{H}), 5.73(\mathrm{t}, J=1.7 \mathrm{~Hz}, 1 \mathrm{H}), 5.09-5.01(\mathrm{~m}, 2 \mathrm{H})$, 2.54 (ddd, $J=12.6,4.9,2.4 \mathrm{~Hz}, 1 \mathrm{H}$ ), 2.49 (dd, $J=14.1,4.8 \mathrm{~Hz}, 1 \mathrm{H}), 2.45$ (dd, $J=5.3,2.1$ $\mathrm{Hz}, 1 \mathrm{H}), 2.44-2.38(\mathrm{~m}, 1 \mathrm{H}), 2.22-2.20(\mathrm{~m}, 1 \mathrm{H}), 2.20-2.17(\mathrm{~m}, 1 \mathrm{H}), 2.16(\mathrm{dd}, J=11.8$, $6.7 \mathrm{~Hz}, 1 \mathrm{H}), 2.11(\mathrm{dt}, J=13.2,3.2 \mathrm{~Hz}, 1 \mathrm{H}), 1.98(\mathrm{td}, J=14.1,5.4 \mathrm{~Hz}, 1 \mathrm{H}), 1.88-1.78(\mathrm{~m}$, 2H), 1.70 (qd, $J=13.3,4.0 \mathrm{~Hz}, 1 \mathrm{H}), 1.62-1.56(\mathrm{~m}, 1 \mathrm{H}), 1.52-1.49(\mathrm{~m}, 1 \mathrm{H}), 1.49-1.47$ $(\mathrm{m}, 1 \mathrm{H}), 1.44-1.37(\mathrm{~m}, 2 \mathrm{H}), 1.30-1.26(\mathrm{~m}, 1 \mathrm{H}), 1.24(\mathrm{~s}, 3 \mathrm{H}), 0.98(\mathrm{~d}, J=6.5 \mathrm{~Hz}, 3 \mathrm{H})$, $0.92(\mathrm{~d}, J=6.7 \mathrm{~Hz}, 3 \mathrm{H}), 0.88(\mathrm{~d}, J=6.7 \mathrm{~Hz}, 3 \mathrm{H}), 0.84(\mathrm{~d}, J=6.7 \mathrm{~Hz}, 3 \mathrm{H}), 0.66(\mathrm{~s}, 3 \mathrm{H})$.

13C-NMR: $\quad\left(151 \mathrm{MHz}, \mathrm{CDCl}_{3}\right) ; \delta[\mathrm{ppm}]=198.6,174.0,162.6,159.5,134.2,132.3,114.8,113.5,58.3$, 56.5, 47.4, 47.1, 40.5, 39.4, 38.6, 34.7, 34.1, 33.5, 33.3, 27.7, 25.5, 22.7, 20.7, 20.5, 20.1, $20.1,18.8,12.6$.

IR: $\quad\left(\right.$ neat); $\tilde{v}\left[\mathrm{~cm}^{-1}\right]=2954(\mathrm{~m}), 2924(\mathrm{~s}), 2853(\mathrm{~m}), 1721(\mathrm{~m}), 1681(\mathrm{w}), 1623(\mathrm{w}), 1458(\mathrm{w})$, $1377(w), 1339(w), 1141(w)$.

HRMS: (ESI-TOF); $\mathrm{m} / z$ calcd. for $\mathrm{C}_{28} \mathrm{H}_{40} \mathrm{O}_{3} \mathrm{Na}^{+}[\mathrm{M}+\mathrm{Na}]^{+}:$447.2870, found: 447.2870.

Opt. act. $\quad[a]_{D}^{20}=+132.5\left(c=0.16, \mathrm{CHCl}_{3}\right)$. 
3 NMR Comparisons of synthetic 2 and 1
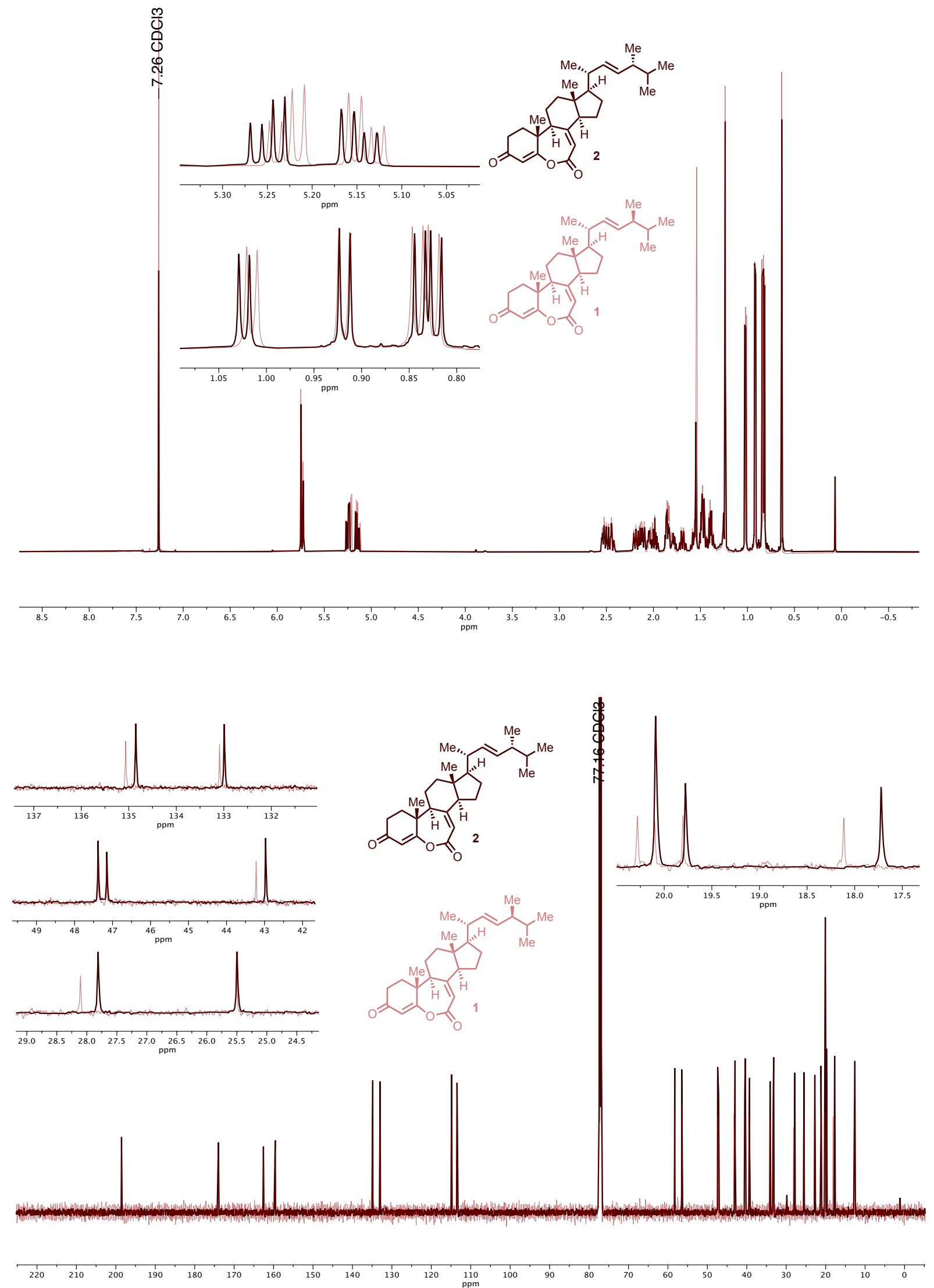
Table S1. ${ }^{1 H}$-NMR comparison of natural materials[11],[9],[12] and synthetic 2 and $\mathbf{1}$.

\begin{tabular}{|c|c|c|c|c|c|c|}
\hline$\#$ & Patent[11],a,b & Herbarulide[9],c & $\Delta \delta_{(\text {Herb. vs. 2) }}$ & Synthetic 2c & Synthetic 1c & Fortisterol[12],a \\
\hline 1 & & $2.19 \mathrm{~m} ; 1.95 \mathrm{~m}$ & $0.00 ; 0.03$ & $\begin{array}{c}2.19 \text { ddd }(14.3,5.0,2.1) \\
1.98 \operatorname{td}(14.2,5.3)\end{array}$ & $\begin{array}{c}2.20 \text { ddd }(14.1,5.0,2.2) \\
1.98 \operatorname{td}(14.3,5.6)\end{array}$ & $2.21 \mathrm{~m} ; 1.96 \mathrm{~m}$ \\
\hline 2 & & $2.46 \mathrm{~m} ; 2.46 \mathrm{~m}$ & $0.03 ; 0.03$ & $\begin{array}{c}2.49 \mathrm{dd}(14.1,4.8) ; 2.43 \\
\operatorname{ddd}(18.4,5.2,1.7)\end{array}$ & $\begin{array}{c}2.49 \mathrm{dd}(14.0,4.8) ; 2.43 \\
\mathrm{ddd}(18.4,5.5,2.0)\end{array}$ & $2.50 \mathrm{~m} ; 1.47 \mathrm{~m}^{*}$ \\
\hline 3 & - & - & - & - & - & - \\
\hline 4 & $5.75 \mathrm{~s}$ & $5.75 \mathrm{~s}$ & 0.00 & $5.75 \mathrm{~s}$ & $5.75 \mathrm{~s}$ & $5.74 \mathrm{~m}$ \\
\hline 5 & - & - & - & - & - & - \\
\hline 6 & - & - & - & - & - & - \\
\hline 7 & $5.73 \mathrm{~s}$ & $5.72 \mathrm{~s}$ & 0.00 & $5.72 \mathrm{t}(1.8)$ & $5.72 \mathrm{t}(1.8)$ & $5.71 \mathrm{~m}$ \\
\hline 8 & - & - & - & - & - & - \\
\hline 9 & & $2.52 \mathrm{~m}$ & 0.01 & $2.53 \mathrm{ddd}(12.4,4.9,2.2)$ & $2.53 \mathrm{~m}$ & $2.51 \mathrm{~m}$ \\
\hline 10 & - & - & - & - & - & - \\
\hline 11 & & $1.84 \mathrm{~m} ; 1.68 \mathrm{~m}$ & $0.02 ; 0.01$ & $\begin{array}{c}1.86 \mathrm{~m}, 1.69 \mathrm{dd}(13.0 \\
4.1)\end{array}$ & $\begin{array}{c}1.85 \mathrm{~m}, 1.69 \mathrm{qd}(13.1 \\
4.1)\end{array}$ & $1.55 \mathrm{~m}^{*} ; 1.52 \mathrm{~m}^{*}$ \\
\hline 12 & & $2.09 \mathrm{~m} ; 1.45 \mathrm{~m}$ & 0.01 & $\begin{array}{c}2.10 \text { ddd }(13.0,4.1,2.8) ; \\
1.48 \mathrm{~m}\end{array}$ & $\begin{array}{c}2.11 \mathrm{dt}(13.1,3.6) ; 1.47 \\
\mathrm{~m}\end{array}$ & $2.12 \mathrm{~m} ; 1.45 \mathrm{~m}$ \\
\hline 13 & - & - & - & - & - & - \\
\hline 14 & & 2.14 & 0.00 & $2.14 \mathrm{~m}$ & $2.14 \mathrm{dd}(12.0,7.5)$ & $2.13 \mathrm{~m}$ \\
\hline 15 & & $1.50 \mathrm{~m} ; 1.50 \mathrm{~m}$ & $0.06 ; 0.02$ & $1.56 \mathrm{~m} ; 1.48 \mathrm{~m}$ & $1.59 \mathrm{~m}, 1.48 \mathrm{~m}$ & $1.84 \mathrm{~m}^{*} ; 1.82 \mathrm{~m}^{*}$ \\
\hline 16 & & $1.78 \mathrm{~m} ; 1.78 \mathrm{~m}^{\star}$ & $0.01 ; 0.39$ & $1.79 \mathrm{~m} ; 1.39 \mathrm{~m}$ & $1.80 \mathrm{~m}, 1.38 \mathrm{~m}$ & $1.73 \mathrm{~m} ; 1.35 \mathrm{~m}$ \\
\hline 17 & & $1.38 \mathrm{~m}$ & 0.01 & $1.39 \mathrm{~m}$ & $1.41 \mathrm{~m}$ & $1.37 \mathrm{~m}$ \\
\hline 18 & & $0.64 \mathrm{~s}$ & 0.01 & $0.63 \mathrm{~s}$ & $0.64 \mathrm{~s}$ & $0.63 \mathrm{~s}$ \\
\hline 19 & $1.24 \mathrm{~s}$ & $1.24 \mathrm{~s}$ & 0.00 & $1.24 \mathrm{~s}$ & $1.24 \mathrm{~s}$ & $1.23 \mathrm{~s}$ \\
\hline 20 & & $2.03 \mathrm{~m}$ & 0.01 & $2.04 \mathrm{~m}$ & $2.04 \mathrm{~m}$ & $2.04 \mathrm{~m}$ \\
\hline 21 & $1.02 \mathrm{~d}(6.5)$ & $1.02 \mathrm{~d}(6.6)$ & 0.00 & $1.02 \mathrm{~d}(6.6)$ & $1.02 \mathrm{~d}(6.6)$ & $1.01 \mathrm{~s}$ \\
\hline 22 & $5.14 \mathrm{~m}$ & $5.15 \mathrm{dd}(15.2,8.5)$ & 0.00 & $5.15 \mathrm{dd}(15.2,8.6)$ & $5.14 \mathrm{dd}(15.2,8.6)$ & $\begin{array}{c}5.25 \text { dd }(15.2 \\
7.7)^{\star}\end{array}$ \\
\hline 23 & $5.25 \mathrm{~m}$ & $5.25 \mathrm{dd}(15.2,7.8)$ & 0.00 & $5.25 \mathrm{dd}(15.2,7.9)$ & $5.23 \mathrm{dd}(15.2,8.1)$ & $\begin{array}{c}5.15 \mathrm{dd}(15.2 \\
8.4)^{\star}\end{array}$ \\
\hline 24 & & $1.86 \mathrm{~m}$ & 0.00 & $1.86 \mathrm{~m}$ & $1.84 \mathrm{~m}$ & $1.84 \mathrm{~m}$ \\
\hline 25 & & $1.48 \mathrm{~m}$ & 0.00 & $1.48 \mathrm{~m}$ & $1.47 \mathrm{~m}$ & $2.44 \mathrm{~m}^{*}$ \\
\hline 26 & $0.84 \mathrm{~d}(8.4)$ & $0.84 \mathrm{~d}(6.8)$ & 0.00 & $0.84 \mathrm{~d}(6.8)$ & $0.84 \mathrm{~d}(6.8)$ & $0.82 \mathrm{~d}(6.8)$ \\
\hline 27 & $0.82 \mathrm{~d}(8.4)$ & $0.82 \mathrm{~d}(6.8)$ & 0.00 & $0.82 \mathrm{~d}(6.8)$ & $0.82 \mathrm{~d}(6.7)$ & $0.82 \mathrm{~d}(6.8)$ \\
\hline 28 & $0.92 \mathrm{~d}(6.8)$ & $0.91 \mathrm{~d}(6.8)$ & 0.00 & $0.91 \mathrm{~d}(6.8)$ & $0.92 \mathrm{~d}(6.8)$ & $0.87 d(6.8)$ \\
\hline
\end{tabular}

All chemical shifts are reported in ppm. Coupling constants are in parentheses and are reported in $\mathrm{Hz}$. $\mathrm{m}=$ centered multiplet. All spectra were measured in $\mathrm{CDCl}_{3}$ and are referenced to the residual solvent peak at $\delta_{\mathrm{H}}=7.26 \mathrm{ppm}$. ${ }^{a}$ Recorded at $400 \mathrm{MHz}$; b signals were not assigned; c recorded at $600 \mathrm{MHz}$, misassigned signals are marked with*. 
Table S2. ${ }^{13} \mathrm{C}-\mathrm{NMR}$ comparison of natural materials[11],[9],[12] and synthetic 2 and $\mathbf{1 .}$

\begin{tabular}{|c|c|c|c|c|c|c|}
\hline \# & Patent[11],a & Herbarulide[9],b & $\Delta \delta_{\text {(Herb. vs. 2) }}$ & Synthetic $2^{b, c}$ & Synthetic $1^{b, c}$ & Fortisterol[12],a \\
\hline 1 & 33.9 & 34.0 & 0.1 & 33.9 & 33.9 & 33.8 \\
\hline 2 & $33.2^{*}$ & 33.2 & 0.0 & 33.2 & 33.2 & $33.1^{*}$ \\
\hline 3 & 198.5 & 198.4 & 0.0 & 198.4 & 198.4 & 198.5 \\
\hline 4 & 114.7 & 114.7 & 0.0 & 114.7 & 114.7 & 114.6 \\
\hline 5 & 173.8 & 173.9 & 0.1 & 173.8 & 173.8 & 173.9 \\
\hline 6 & 162.4 & 162.4 & 0.0 & 162.4 & 162.4 & 162.4 \\
\hline 7 & 113.3 & 113.4 & 0.1 & 113.3 & 113.3 & 113.2 \\
\hline 8 & 159.5 & 159.4 & 0.0 & 159.4 & 159.4 & 159.5 \\
\hline 9 & 47.2 & 47.3 & 0.1 & 47.2 & 47.2 & 47.2 \\
\hline 10 & 40.3 & 40.4 & 0.0 & 40.4 & 40.4 & 40.3 \\
\hline 11 & $22.6^{\star}$ & 25.4 & 0.1 & 25.3 & 25.3 & $22.6^{\star}$ \\
\hline 12 & 39.2 & 39.2 & 0.1 & 39.1 & 39.2 & 39.1 \\
\hline 13 & 45.9 & 47.0 & 0.0 & 47.0 & 47.0 & 47.0 \\
\hline 14 & 58.1 & 58.2 & 0.1 & 58.1 & 58.1 & 58.0 \\
\hline 15 & $25.3^{\star}$ & 22.6 & 0.0 & 22.6 & 22.6 & $25.3^{*}$ \\
\hline 16 & 27.7 & 27.7 & 0.0 & 27.7 & 27.9 & 27.6 \\
\hline 17 & 56.3 & 56.4 & 0.1 & 56.3 & 56.3 & 56.3 \\
\hline 18 & 12.4 & 12.5 & 0.1 & 12.4 & 12.4 & 12.4 \\
\hline 19 & 19.9 & 20.0 & 0.1 & 19.9 & 19.9 & 19.9 \\
\hline 20 & 40.3 & 40.2 & 0.0 & 40.2 & 40.3 & 40.2 \\
\hline 21 & 21.0 & 21.1 & 0.1 & 21.0 & 21.0 & 21.0 \\
\hline 22 & 134.7 & 134.7 & 0.0 & 134.7 & 134.9 & 134.7 \\
\hline 23 & 132.8 & 132.9 & 0.1 & 132.8 & 132.9 & 132.8 \\
\hline 24 & 42.8 & 42.9 & 0.1 & 42.8 & 43.1 & 42.8 \\
\hline 25 & $33.0^{\star}$ & 33.1 & 0.1 & 33.0 & 33.1 & $33.0^{*}$ \\
\hline 26 & 19.6 & 20.0 & 0.1 & 19.9 & 20.1 & 19.9 \\
\hline 27 & 19.9 & 19.7 & 0.1 & 19.6 & 19.6 & 19.6 \\
\hline 28 & 17.6 & 17.6 & 0.0 & 17.6 & 18.0 & 17.5 \\
\hline
\end{tabular}

All chemical shifts are reported in ppm. ${ }^{a}$ Recorded at $100 \mathrm{MHz}$; b recorded at $151 \mathrm{MHz}$; ${ }^{\mathrm{b}}$ spectra referenced to the solvent peak at $\delta_{C}=77.00 \mathrm{ppm}$, misassigned signals are marked with ${ }^{*}$. Signals that are shifted due to different configuration at $\mathrm{C} 24$ are marked in light grey. 


\section{NMR Spectra}
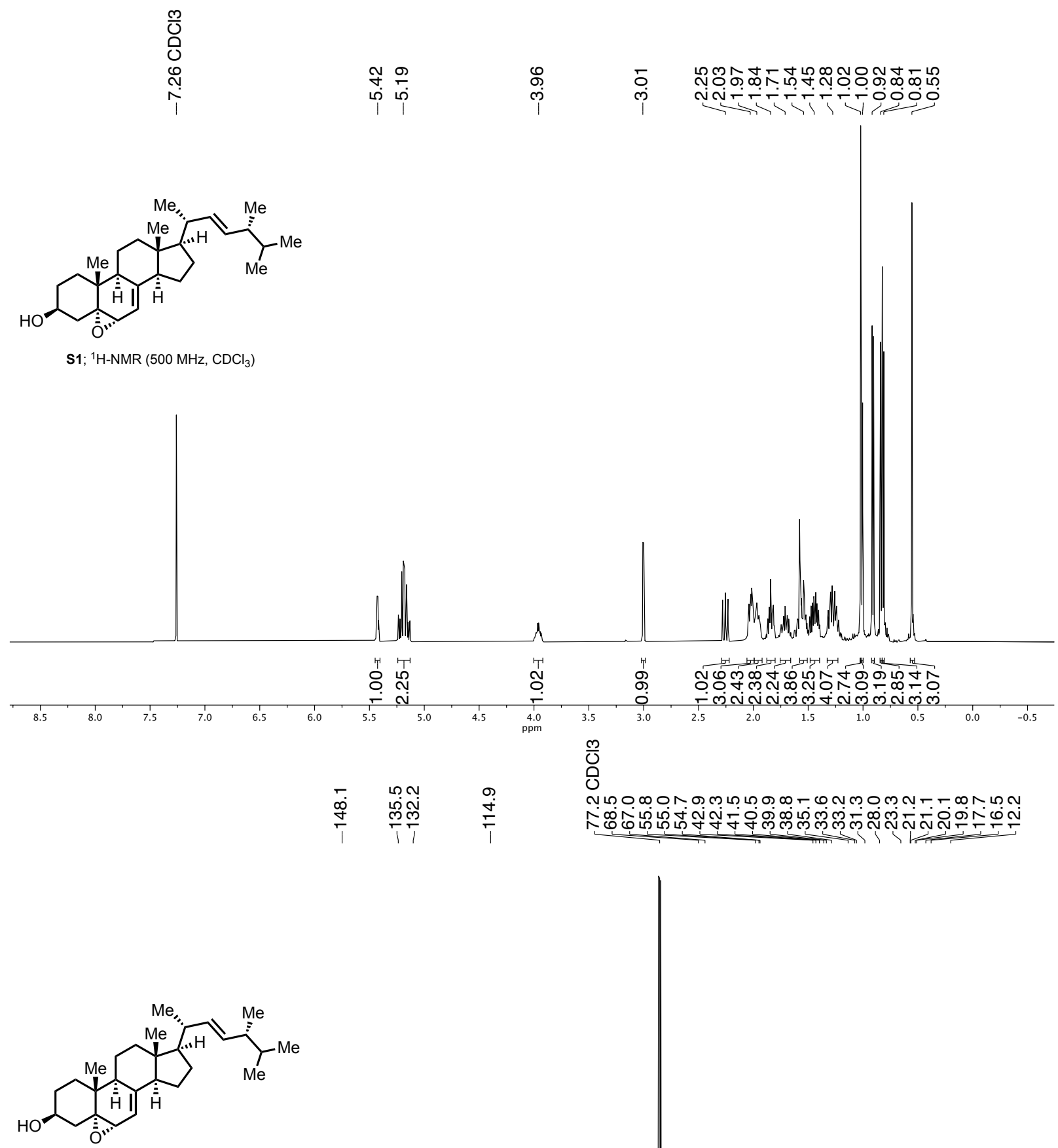

S1; ${ }^{13} \mathrm{C}-\mathrm{NMR}\left(126 \mathrm{MHz}, \mathrm{CDCl}_{3}\right)$
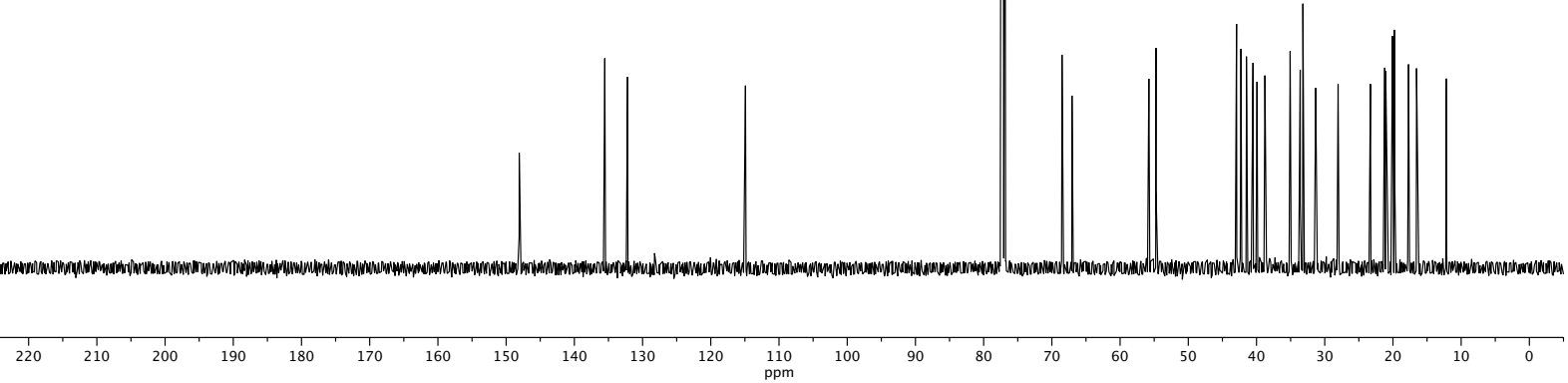

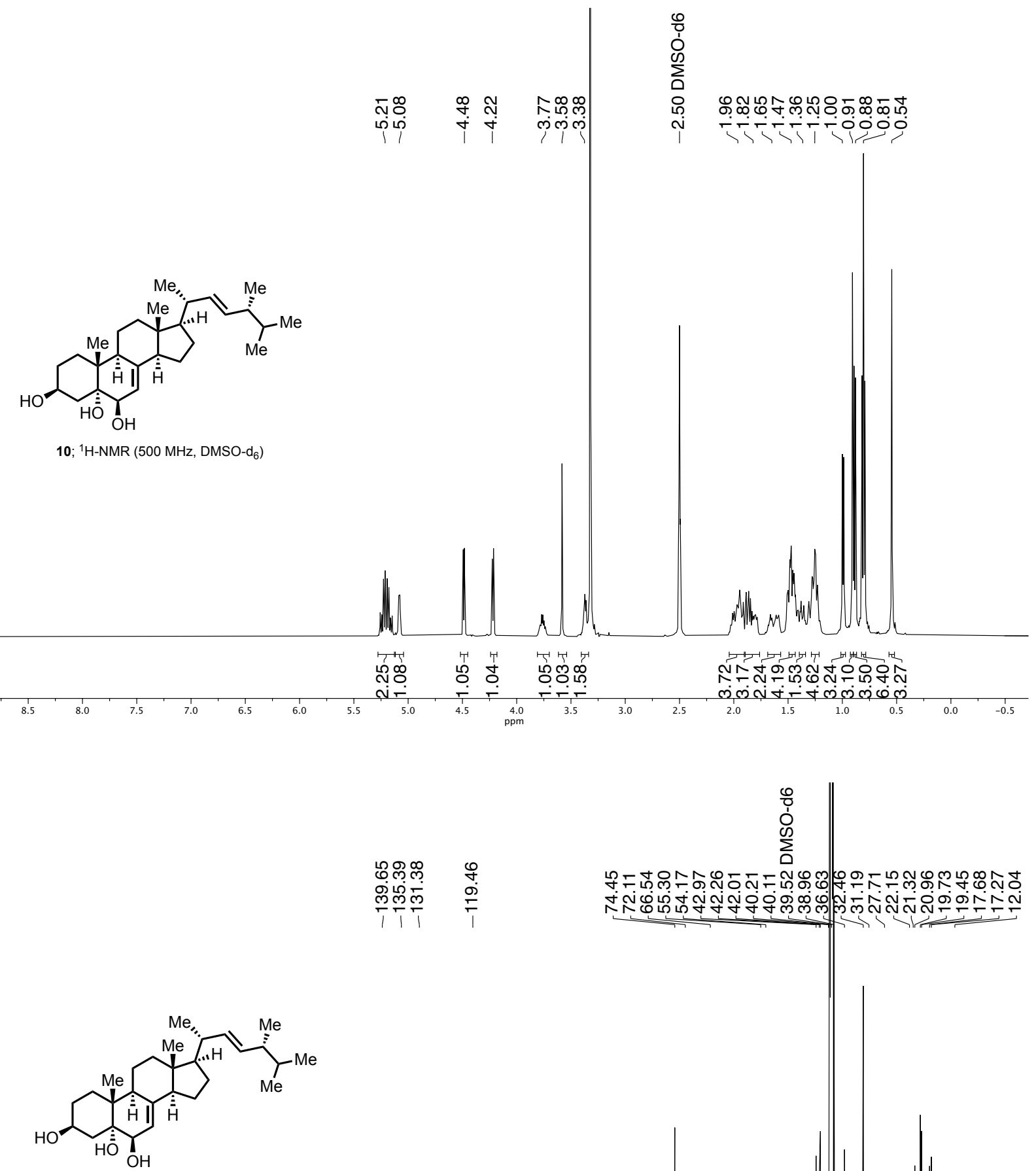

10; ${ }^{13} \mathrm{C}-\mathrm{NMR}\left(126 \mathrm{MHz}\right.$, DMSO-d $\left.\mathrm{d}_{6}\right)$

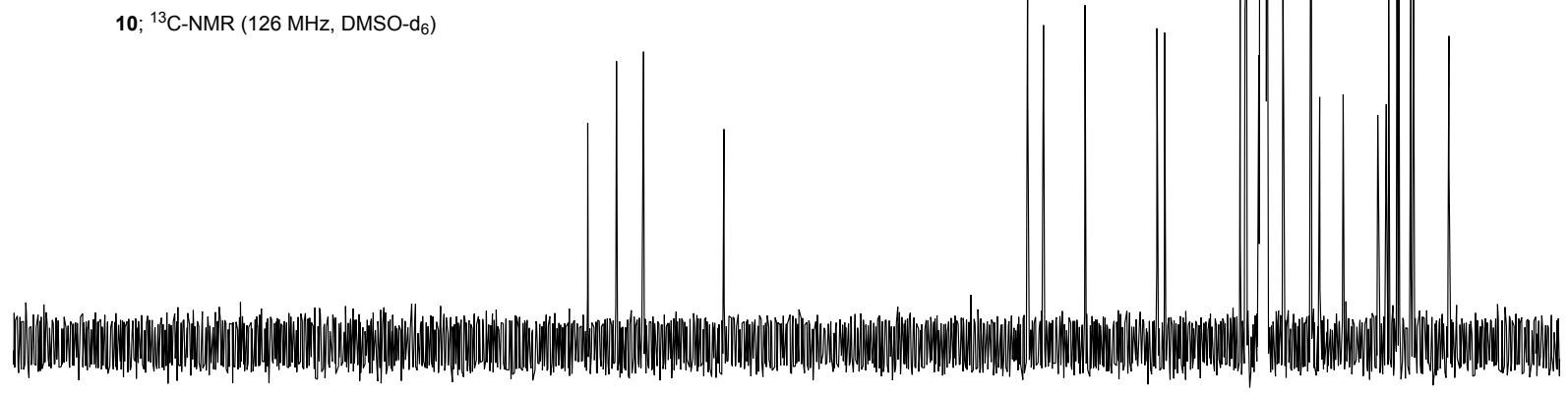

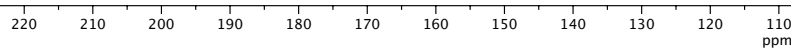




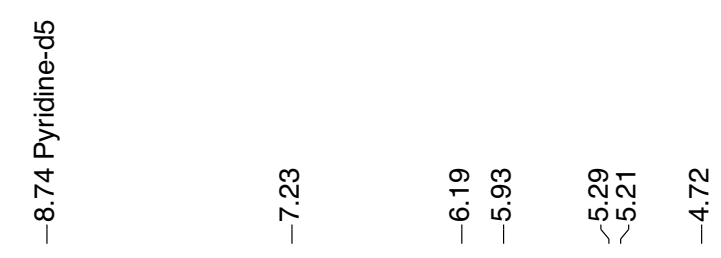

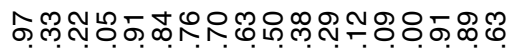

NNNNT-K-T-T-T00

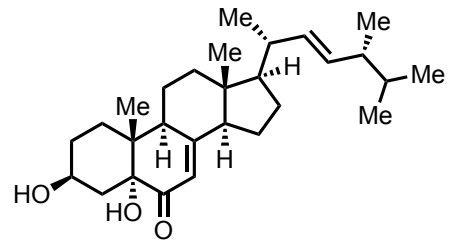

S2; ${ }^{1} \mathrm{H}-\mathrm{NMR}\left(500 \mathrm{MHz}\right.$, pyridine- $\left.\mathrm{d}_{5}\right)$
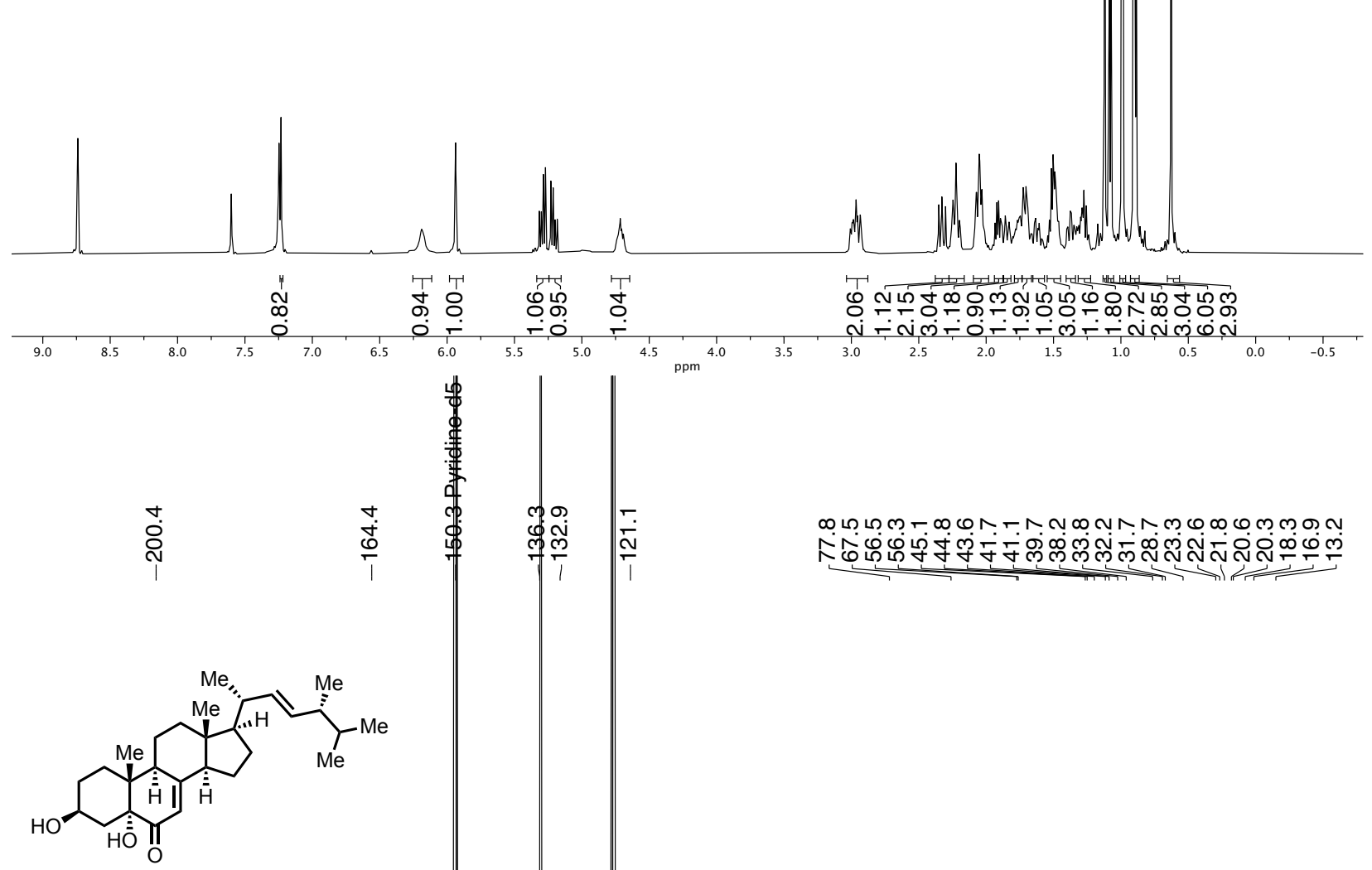

S2; ${ }^{13} \mathrm{C}-\mathrm{NMR}\left(126 \mathrm{MHz}\right.$, pyridine- $\left.\mathrm{d}_{5}\right)$

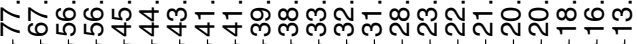

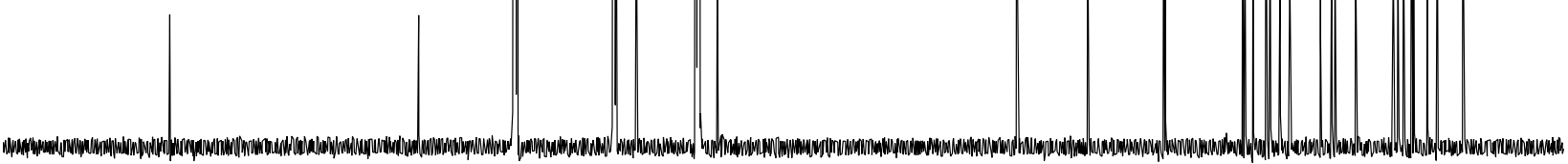

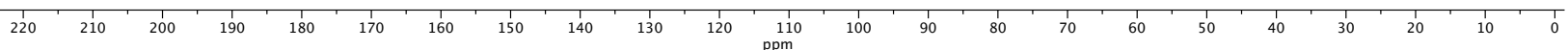




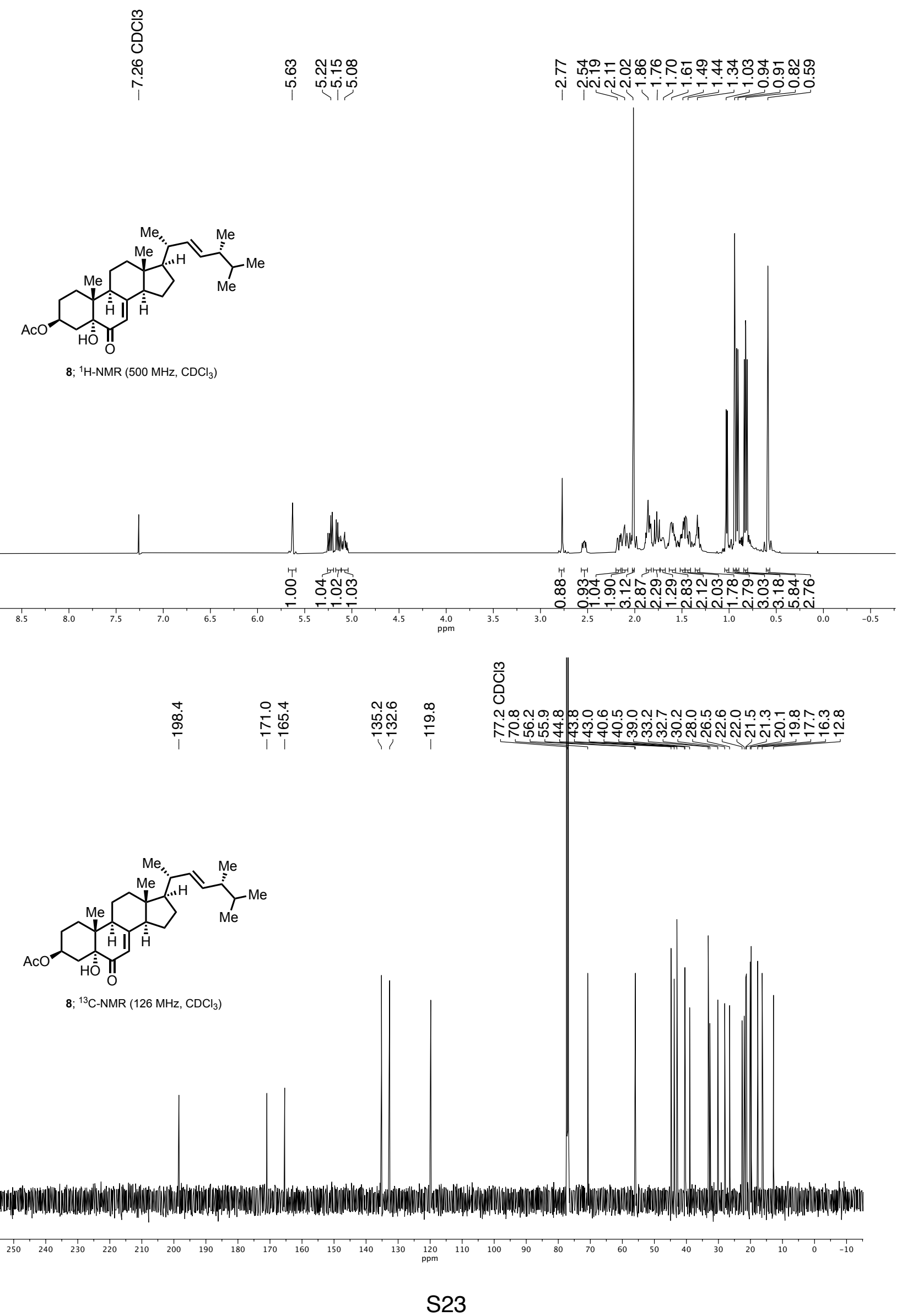


$m$
0
0
0
$\stackrel{0}{1}$
$i$

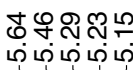

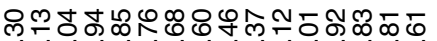

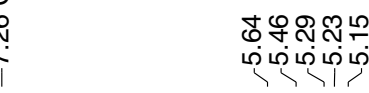

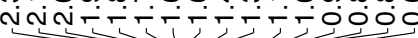

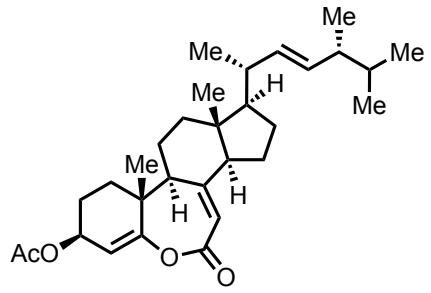

11; ${ }^{1} \mathrm{H}-\mathrm{NMR}\left(600 \mathrm{MHz}, \mathrm{CDCl}_{3}\right.$ )
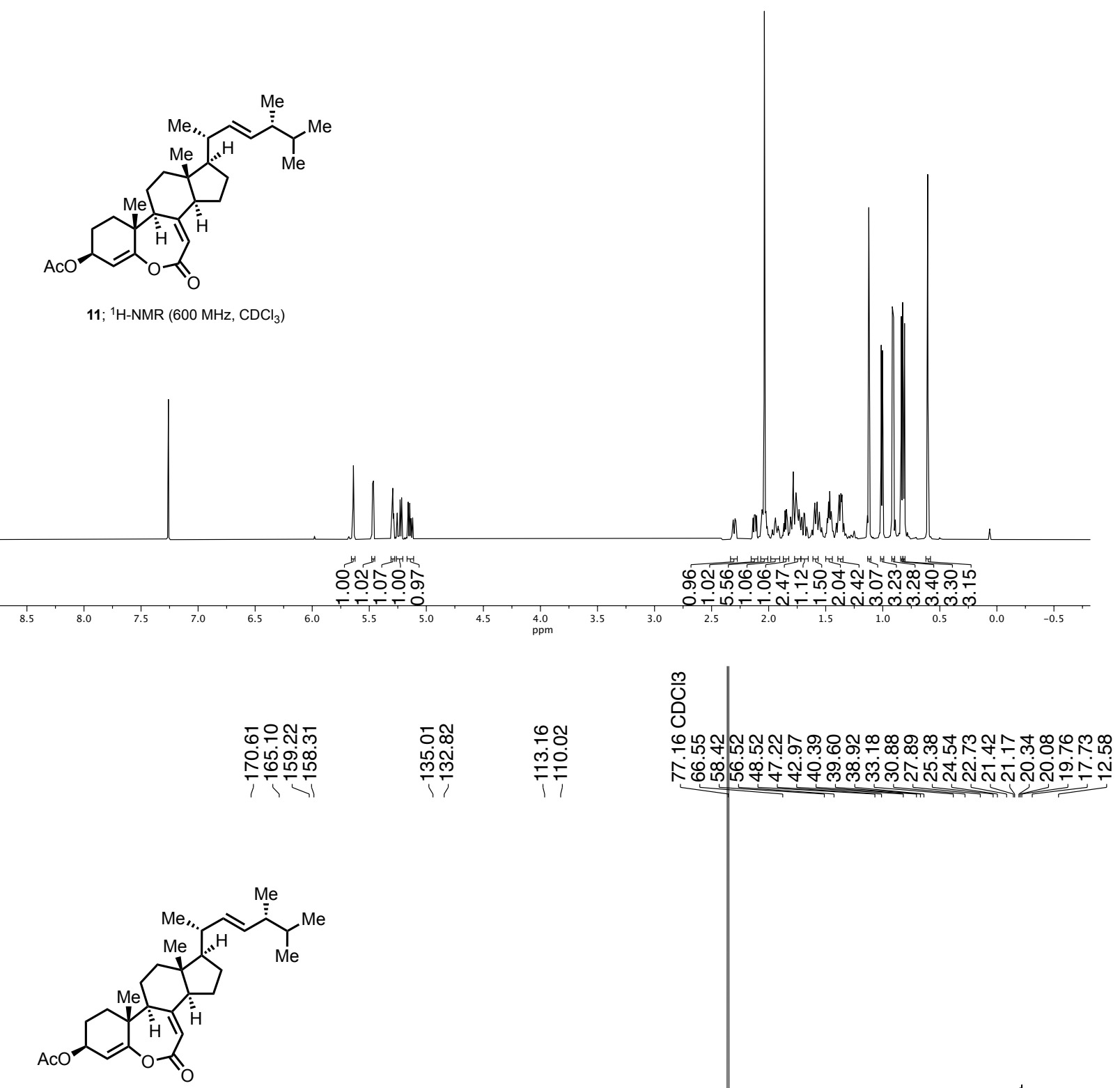

11; ${ }^{13} \mathrm{C}-\mathrm{NMR}\left(151 \mathrm{MHz}, \mathrm{CDCl}_{3}\right.$ )

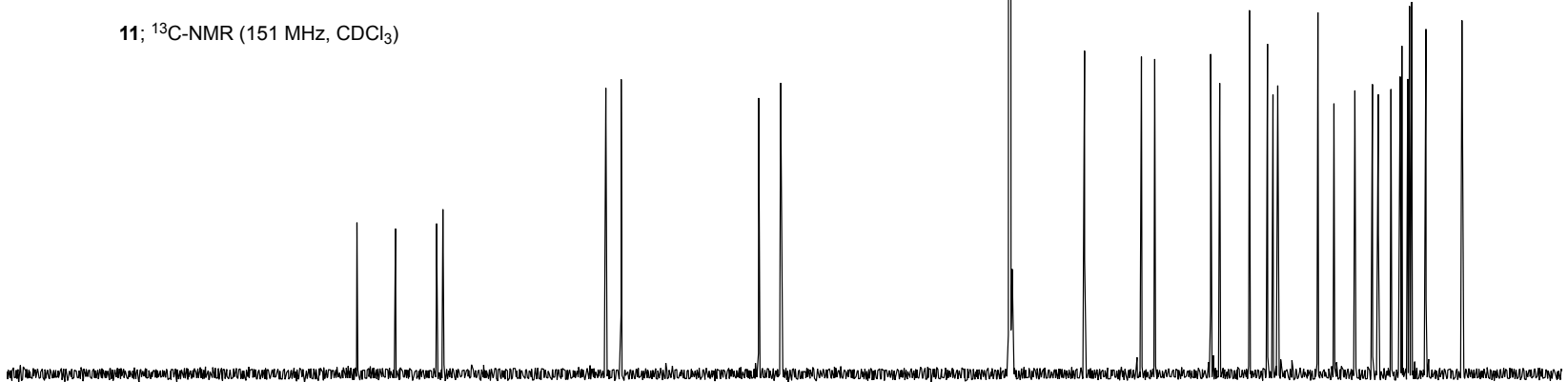

$\frac{1}{20}$

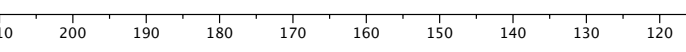

110
$\mathrm{ppm}$ 


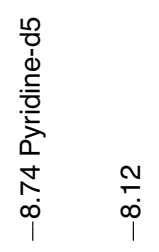

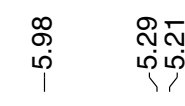

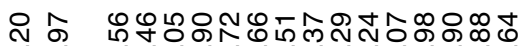

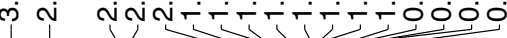

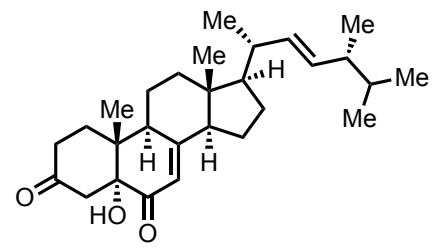

7; ${ }^{1} \mathrm{H}-\mathrm{NMR}\left(500 \mathrm{MHz}\right.$, pyridine- $\mathrm{d}_{5}$ )
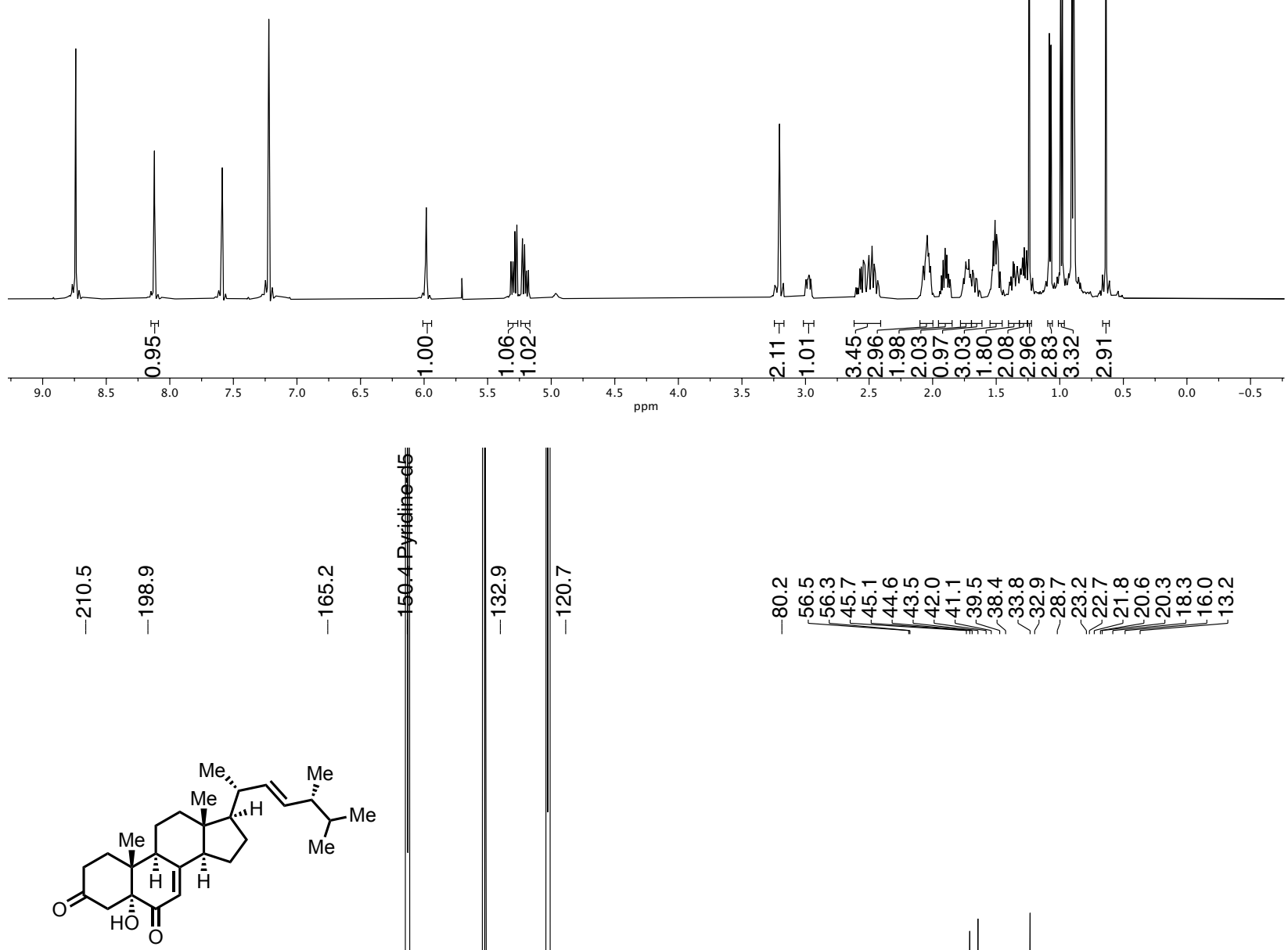

7; ${ }^{13} \mathrm{C}-\mathrm{NMR}\left(126 \mathrm{MHz}\right.$, pyridine-d $\mathrm{d}_{5}$ )

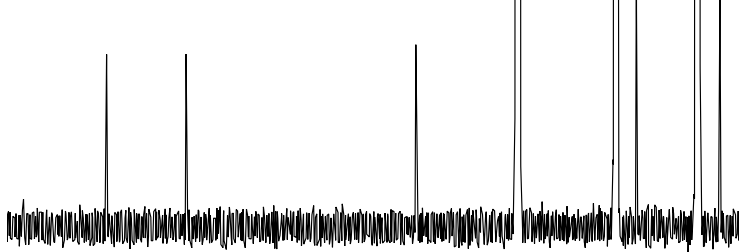

กุ เ ต

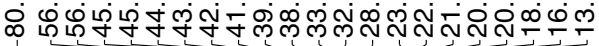
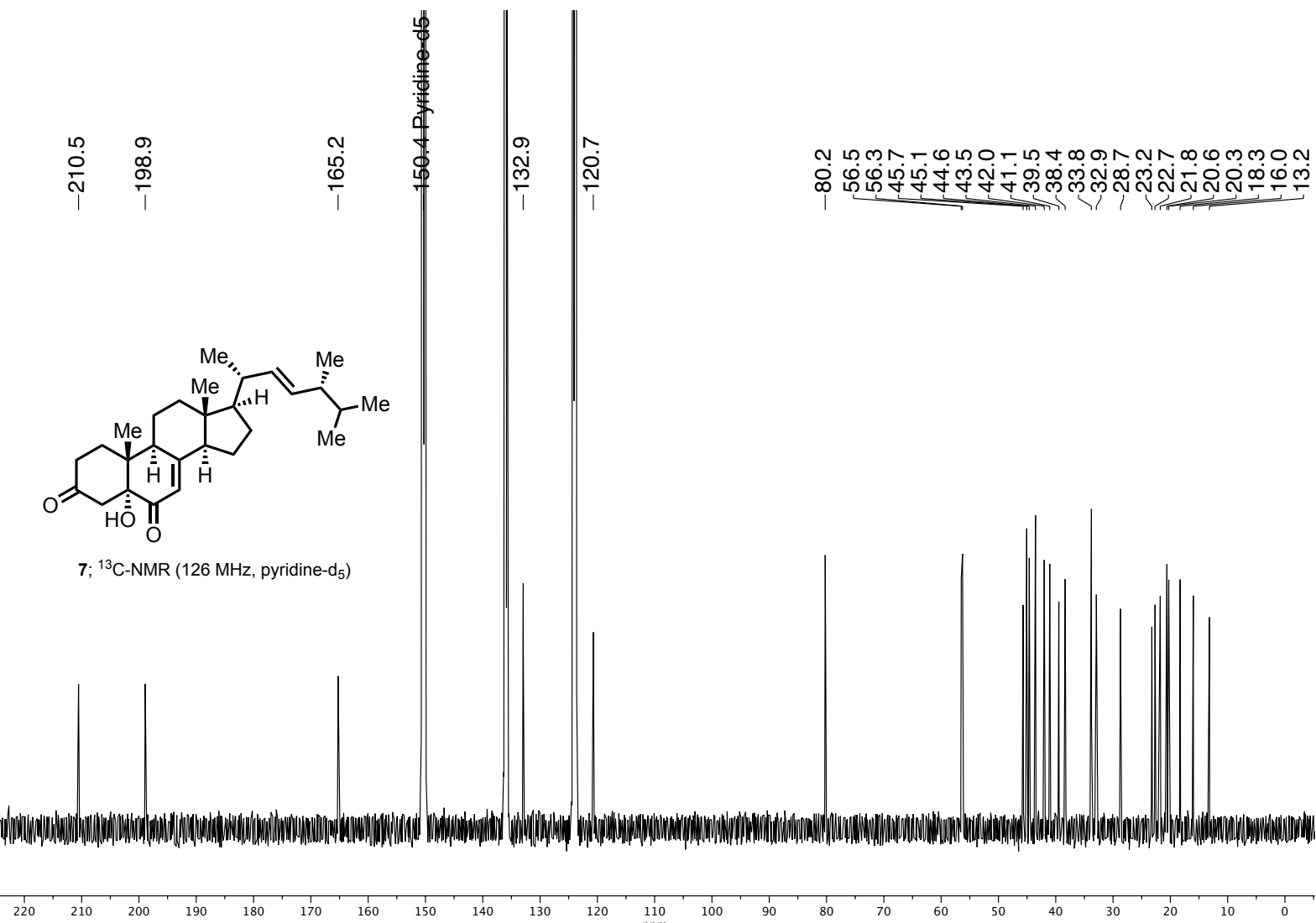


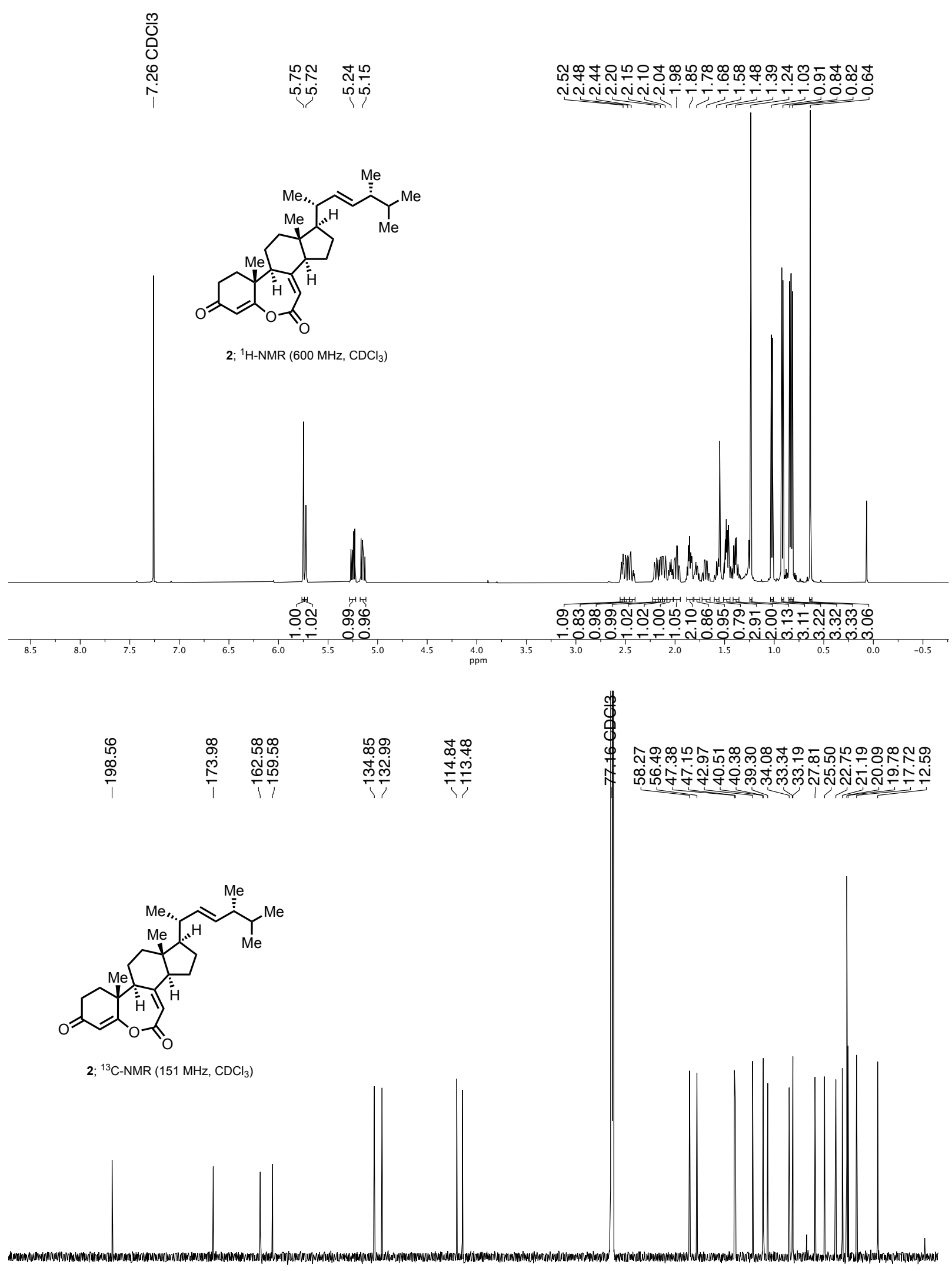

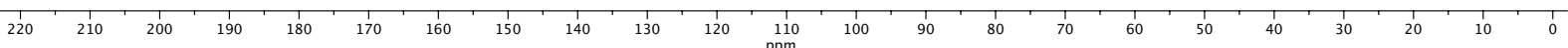



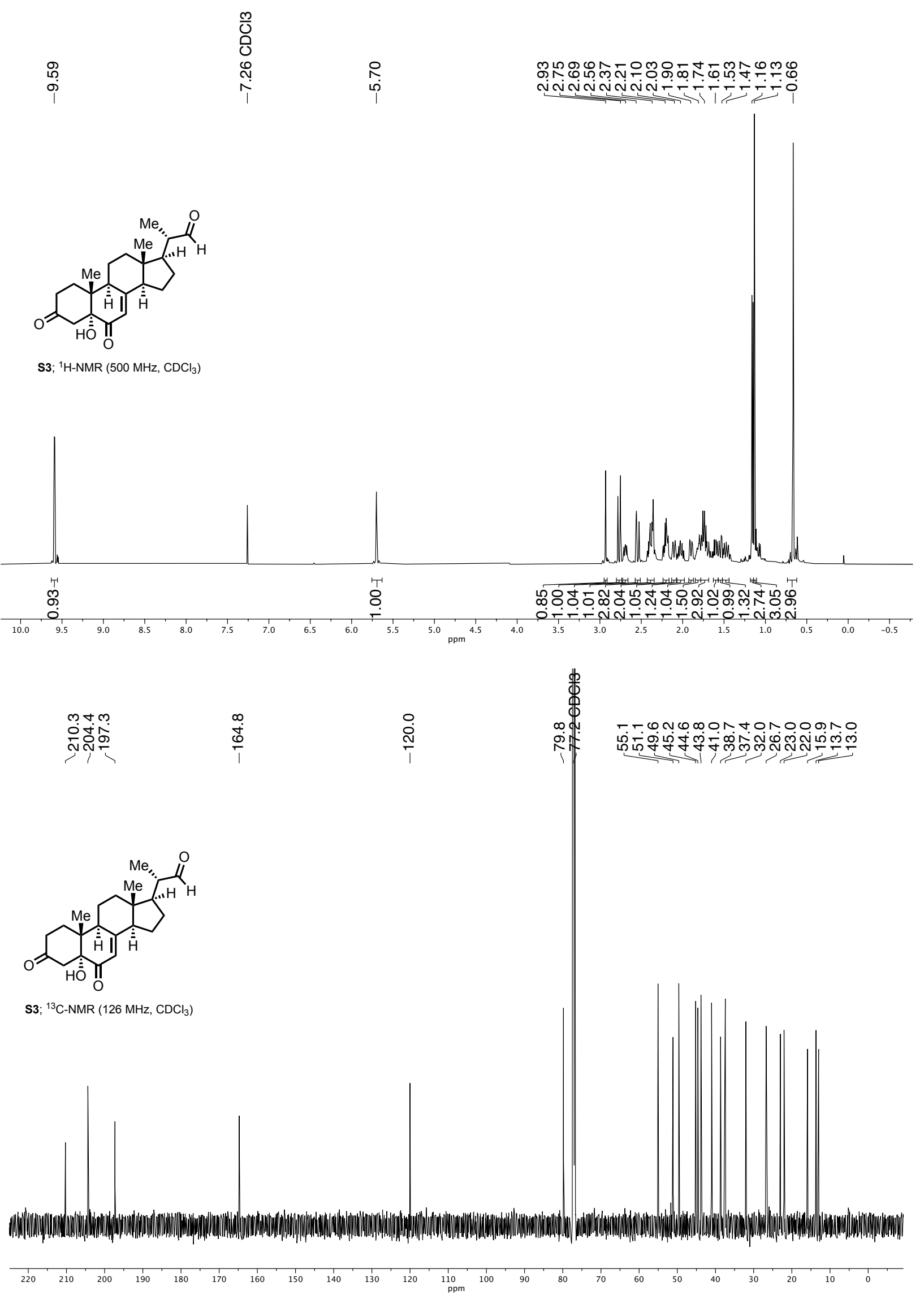


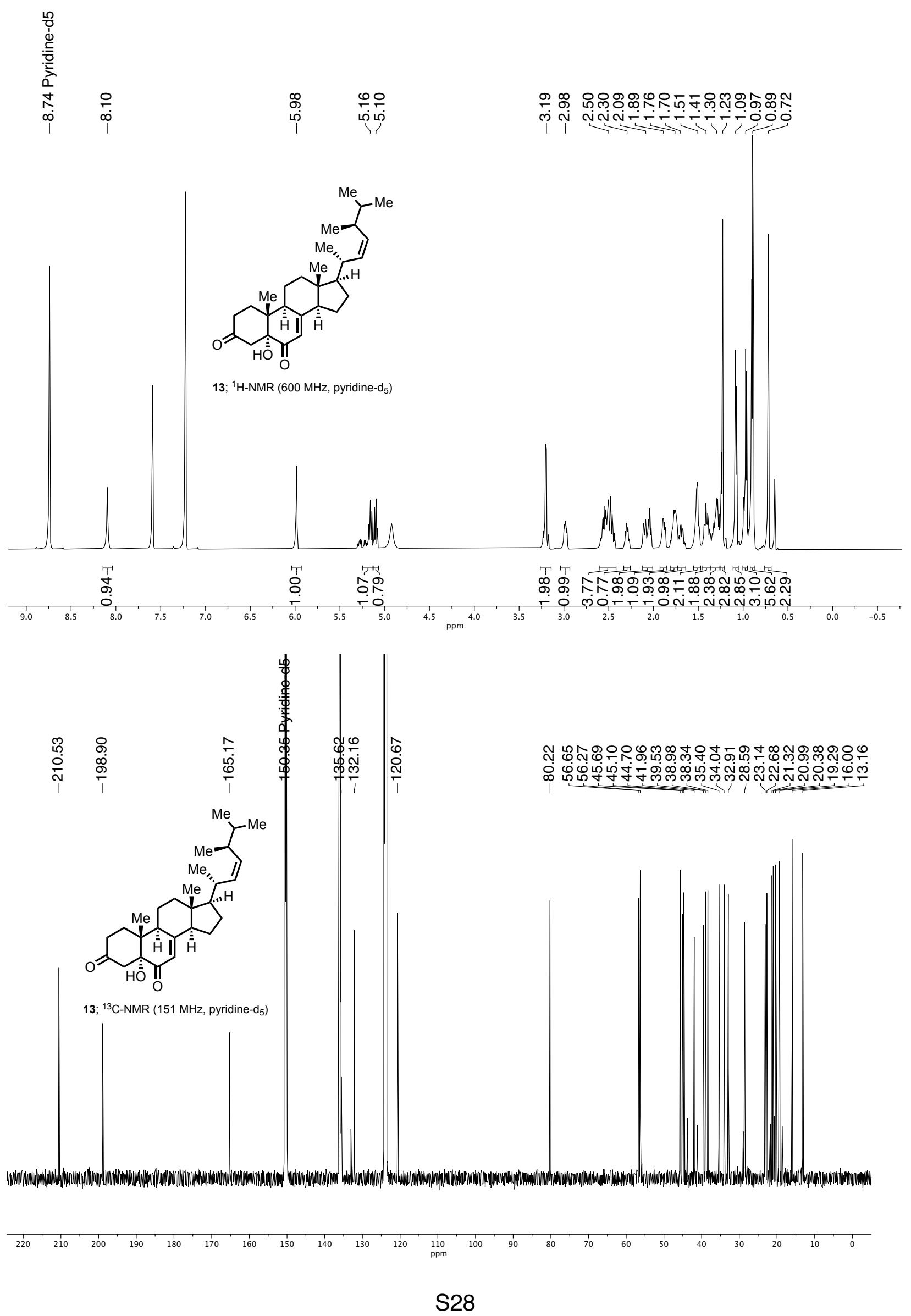



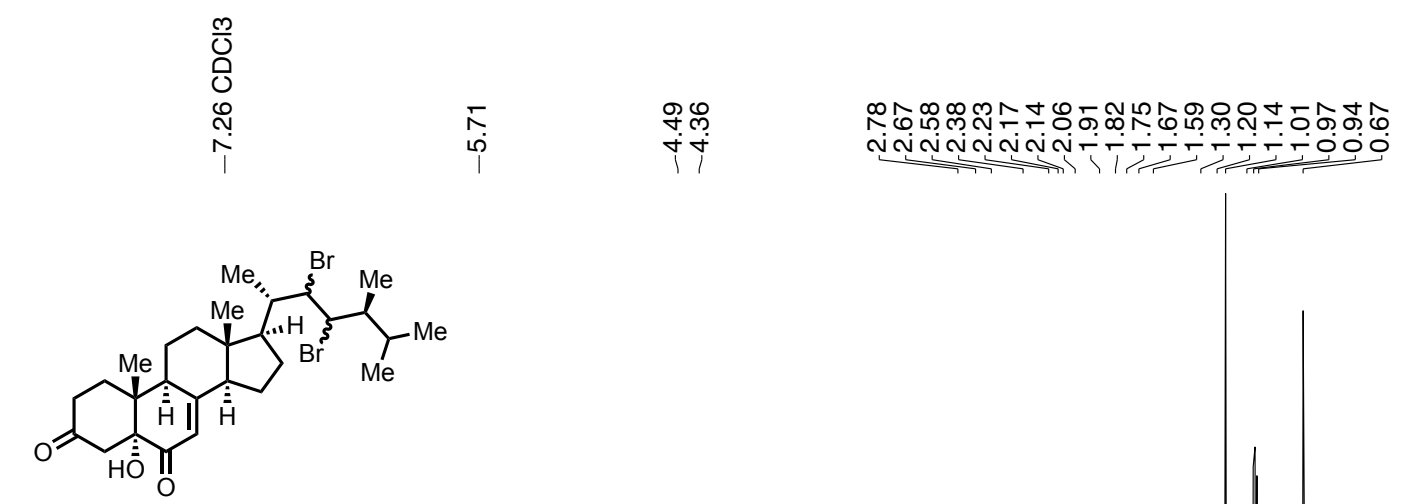

S4 (d.r. 2:1); ${ }^{1} \mathrm{H}-\mathrm{NMR}\left(600 \mathrm{MHz}, \mathrm{CDCl}_{3}\right)$
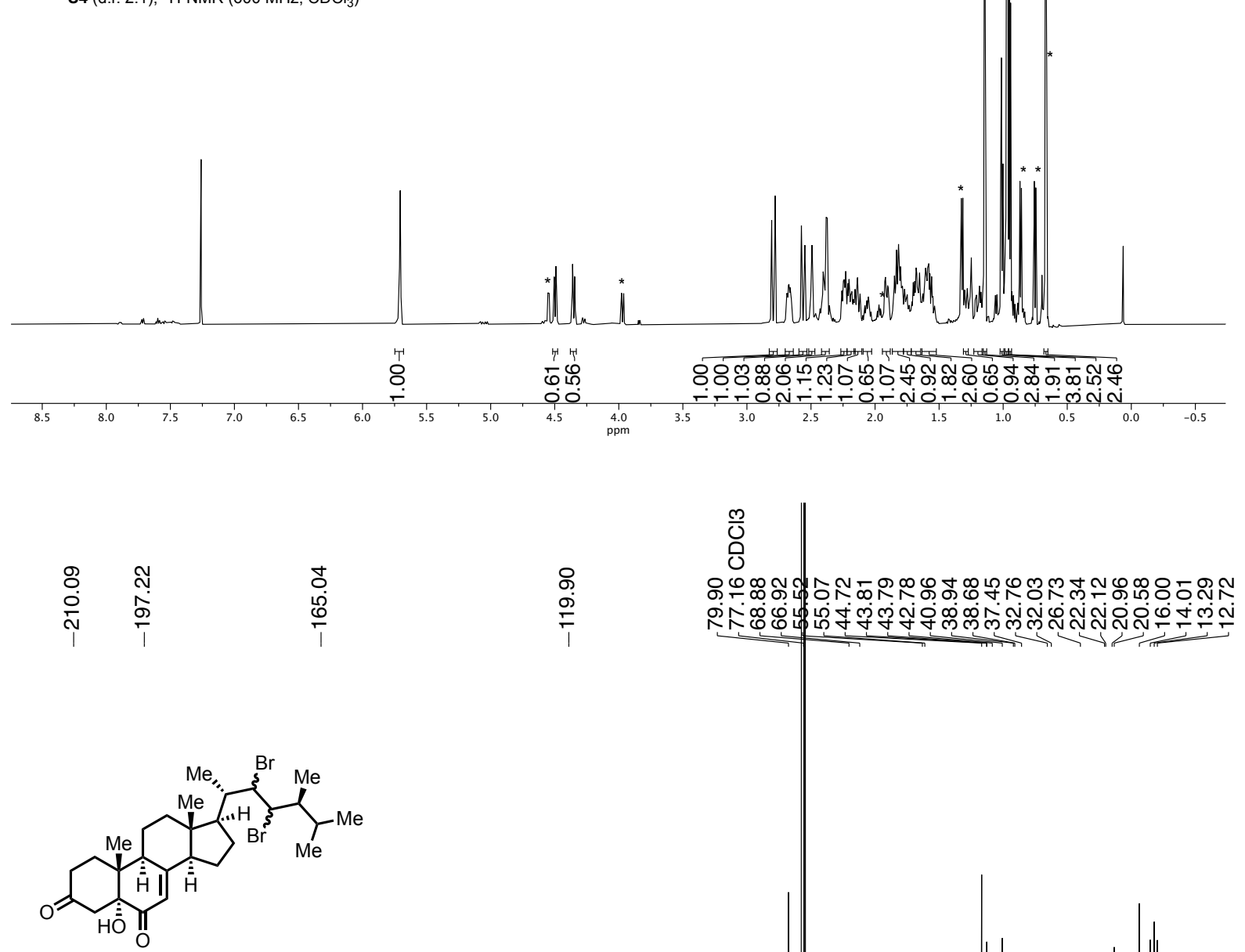

S4 (d.r. $2: 1) ;{ }^{13} \mathrm{C}-\mathrm{NMR}\left(151 \mathrm{MHz}, \mathrm{CDCl}_{3}\right.$ )
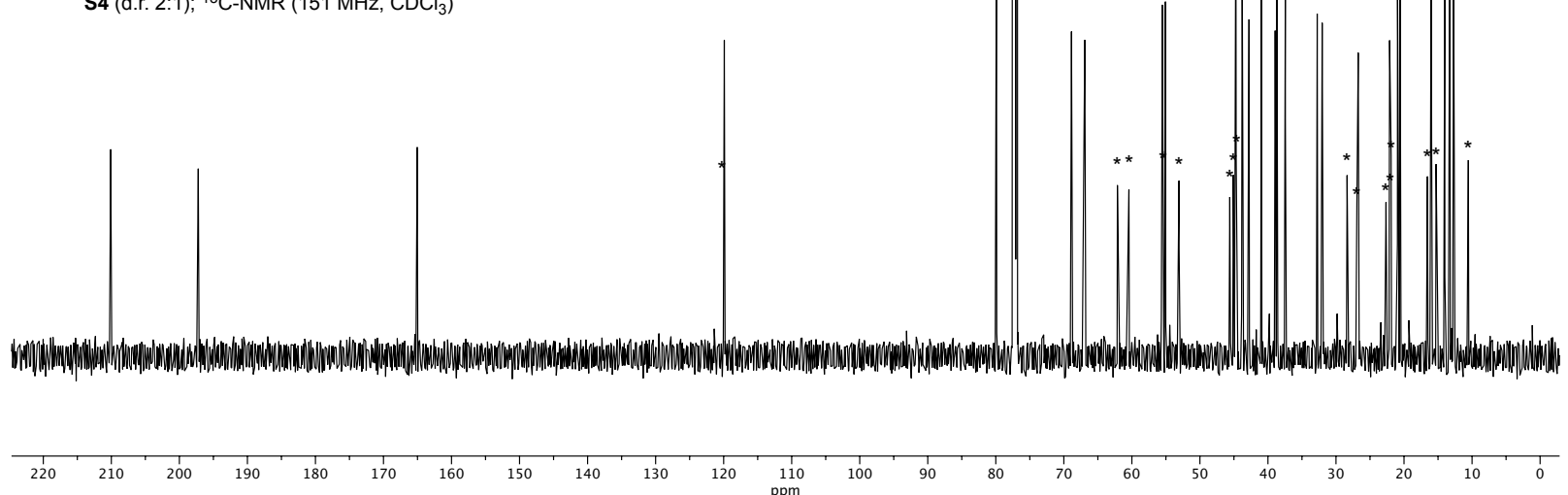

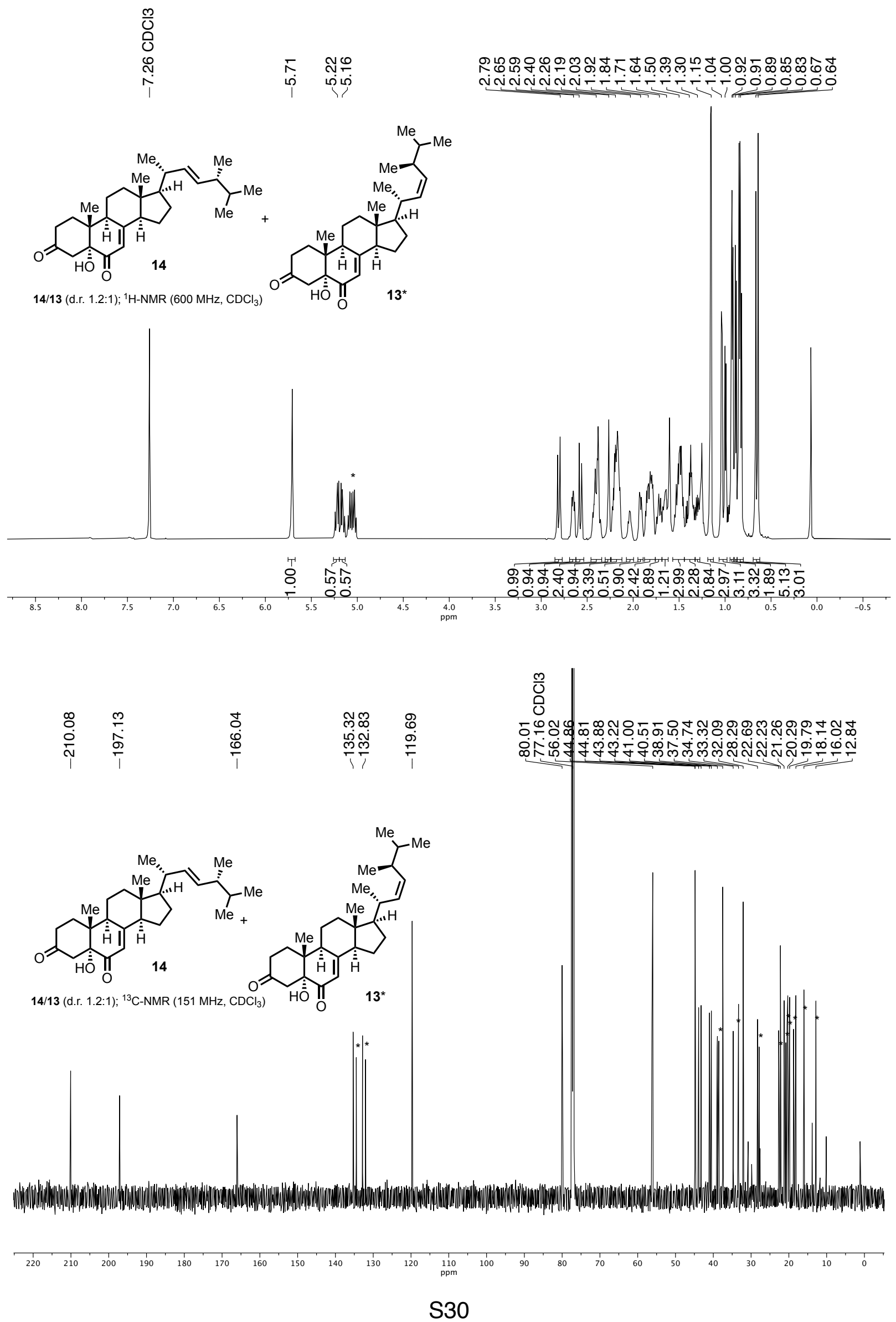


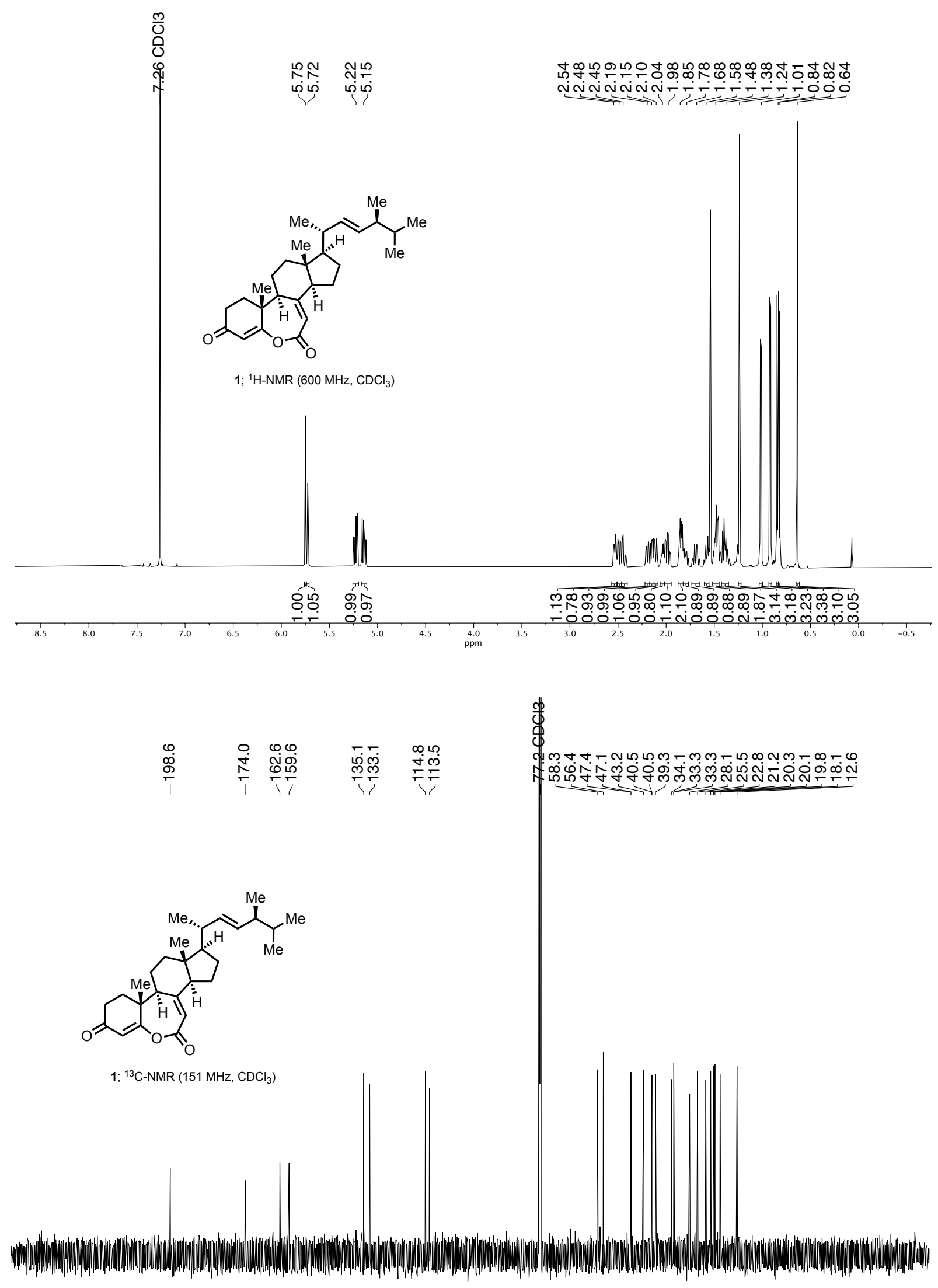

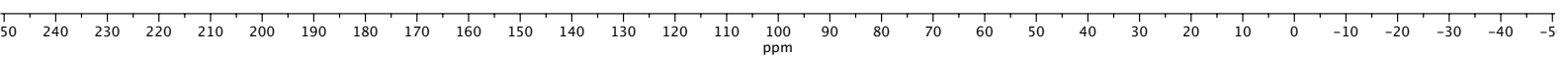



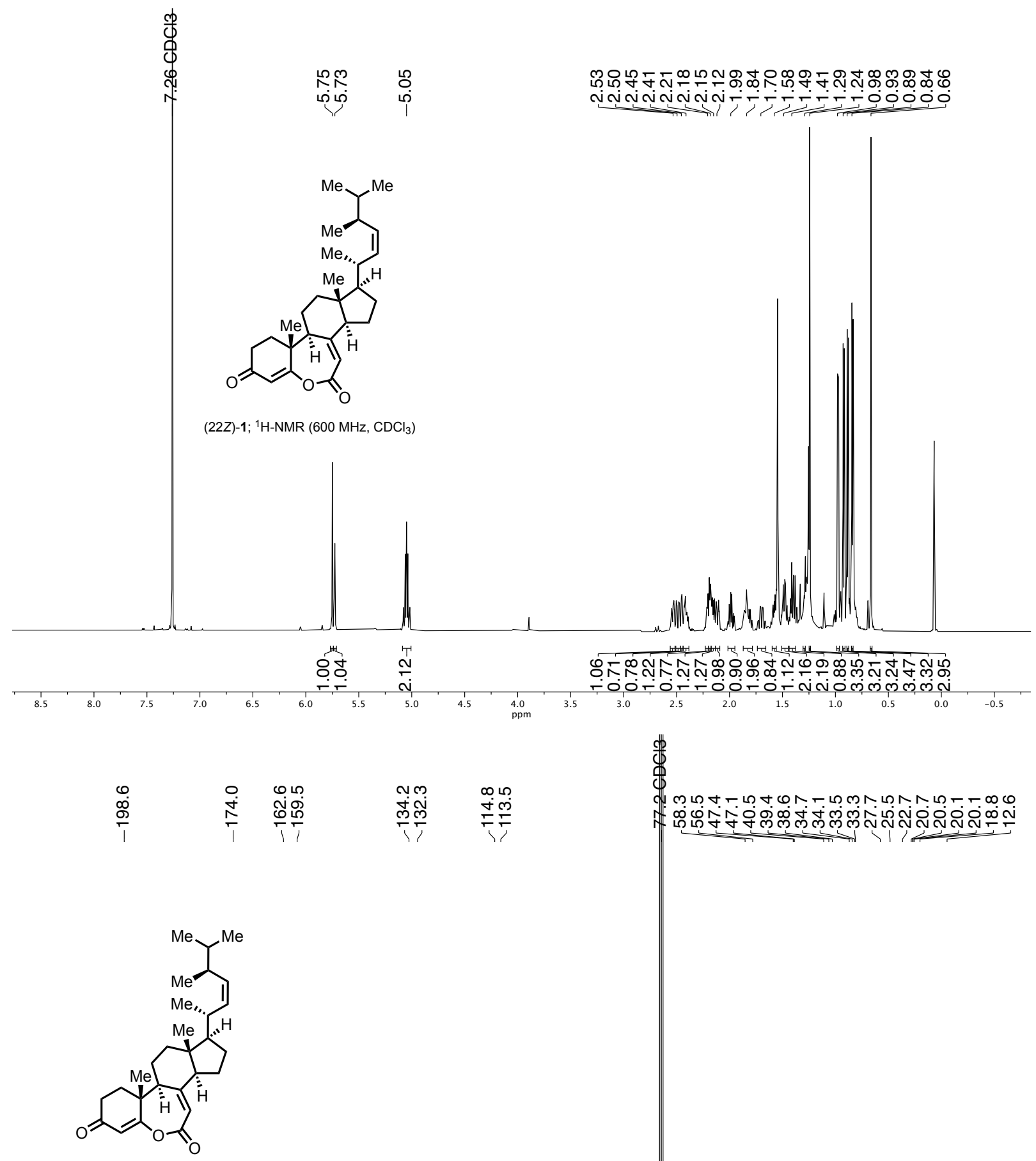

(22Z)-1; ${ }^{13} \mathrm{C}-\mathrm{NMR}\left(151 \mathrm{MHz}, \mathrm{CDCl}_{3}\right)$

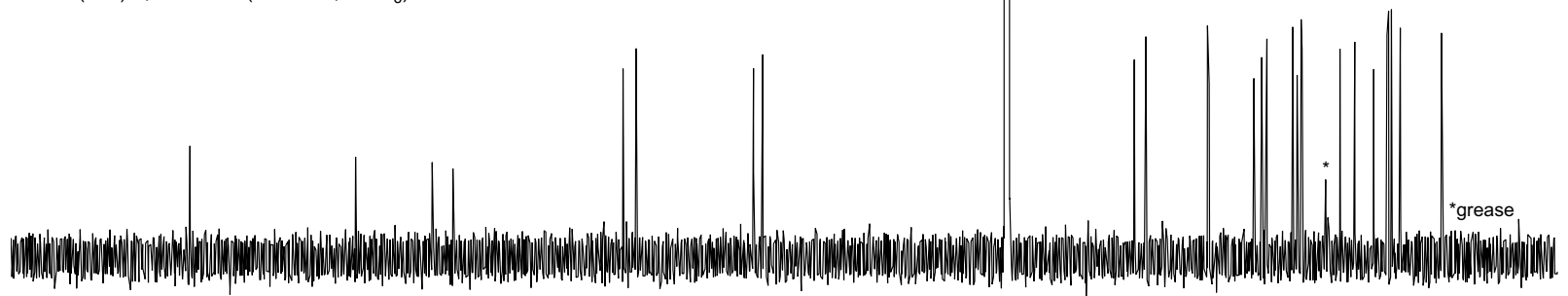

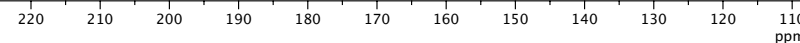




\section{References}

[1] J. W. Bok, L. Lermer, J. Chilton, H. G. Klingeman, G. H. N. Towers, Antitumor sterols from the mycelia of Cordyceps sinensis. Phytochemistry 1999, 51, 891-898.

[2] S. H. Lee, S. H. Shim, J. S. Kim, S. S. Kang, Constituents from the Fruiting Bodies of Ganoderma applanatum and Their Aldose Reductase Inhibitory Activity. Arch. Pharm. Res. 2006, 29, 479483.

[3] E. M. Honeywell, C. E. Bills, Cerevisterol, a sterol accompanying ergosterol in yeast. J. Biolog. Chem. 1932, 99, 71-78.

[4] J. Valisolalao, B. Luu, G. Ourisson, STEROIDES CYTOTOXIQUES DE POLYPORUS VERSICOLOR. Tetrahedron 1983, 39, 2779-2785.

[5] S.-P. Yang, J. Xu, J.-M. Yue, Sterols from the Fungus Catathelasma imperiale. Chinese J. Chem. 2003, 21, 1390-1394.

[6] C. Brosa, R. Puig, X. Comas, C. Fernández, New synthetic strategy for the synthesis of 24epibrassinolide. Steroids 1996, 61, 540-543.

[7] M. Fieser, A. Quilico, A. Nickon, W. E. Rosen, E. J. Tarlton, L. F. Fieser, Permanganate Oxidation of Ergosterol. J. Am. Chem. Soc. 1953, 75, 4066-4071.

[8] G. Kusano, Y. Koike, H. Inoue, S. Nozoe, The Constituents of Gymnopilus spectabilis. Chem. Pharm. Bull. 1986, 34, 3465-3470.

[9] K. Krohn, C. Biele, H.-J. Aust, S. Draeger, B. Schulz, Herbarulide, a Ketodivinyllactone Steroid with an Unprecedented Homo-6-oxaergostane Skeleton form the Endophytic Fungus Pleospora herbarum. J. Nat. Prod. 1999, 62, 629-630.

[10] F. L. Duecker, R. C. Heinze, P. Heretsch, Synthesis of Swinhoeisterol A, Dankasterone A and B, and Periconiastone A by Radical Framework Reconstruction. J. Am. Chem. Soc. 2020, 142, 104-108.

[11] D. Chen, M. Fan, Sterol Lactol Compound and its application as complement inhibitor. Faming Zhuanli Shenqing CN 105985403 A, October 5, 2016.

[12] X.-C. Huang, Y.-W. Guo, G.-Q. Song, Fortisterol, a novel steroid with an unusual sevenmembered lactone ring $B$ from the Chinese marine sponge Biemna fortis Topsent. J. Asian Nat. Prod. Res. 2006, 8, 485-489. 